Journal for ImmunoTherapy of Cancer

\title{
Immunotherapy combining tumor and endothelium cell lysis with immune enforcement by recombinant MIP-3a Newcastle disease virus in a vessel- targeting liposome enhances antitumor immunity
}

Jin-Yan Wang, ${ }^{1}$ Hengyu Chen, ${ }^{1,2}$ Shu-Zhen Dai, ${ }^{1}$ Feng-Ying Huang, ${ }^{1}$ Ying-Ying Lin, ${ }^{1}$ Cai-Chun Wang, ${ }^{3}$ Lei Li, ${ }^{2}$ Wu-Ping Zheng, ${ }^{1}$ Guang-Hong Tan (D) ${ }^{1}$

\section{ABSTRACT \\ Background Several agents for oncolytic immunotherapy have been approved for clinical use, but monotherapy is modest for most oncolytic agents. The combination of several therapeutic strategies through recombinant and nanotechnology to engineer multifunctional oncolytic viruses for oncolytic immunotherapy is a promising strategy.}

Methods An endothelium-targeting iRGD-liposome encapsulating a recombinant Newcastle disease virus (NDV), which expresses the dendritic cell (DC) chemokine MIP-3 $\alpha$ (iNDV3 $\alpha-$ LP), and three control liposomes were constructed. MIP-3 $\alpha$, HMGB1, IgG, and ATP were detected by western blotting or ELISA. The chemotaxis of DCs was examined by Transwell chambers. The phenotypes of the immune cells were analyzed by flow cytometry. The antitumor efficiency was investigated in B16 and 4T1 tumor-bearing mice. Immunofluorescence and immunohistochemistry were used to observe the localization of liposomes, molecular expression and angiogenesis. Synergistic index was calculated using the data of tumor volume, tumor angiogenesis and tumorinfiltrating lymphocytes.

Results Compared with NDV-LP, treatment with iNDV3 $\alpha$ $\mathrm{LP}$ and NDV3 $\alpha-\mathrm{LP}$ induced stronger virus replication and cell lysis in B16 and 4T1 tumor cells and human umbilical vein endothelial cells (HUVECs) with the best response observed following iNDV3 $\alpha-$ LP treatment. B16 and $4 \mathrm{~T} 1$ cells treated with iNDV3 $\alpha$-LP produced more damage-associated molecular pattern molecules, including secreted HMGB1, ATP, and calreticulin. Moreover, iNDV $3 \alpha-$ LP specifically bound to $\alpha \mathrm{V} \beta 3$-expressing $4 \mathrm{~T} 1$ cells and HUVECs and to tumor neovasculature. Tumor growth was significantly suppressed, and survival was longer in iNDV3 $\alpha$-LP-treated B16-bearing and 4T1-bearing mice. A mechanism study showed that iNDV3 $\alpha$-LP treatment initiated the strongest tumor-specific cellular and humoral immune response. Moreover, iNDV3 $\alpha$-LP treatment could significantly suppress tumor angiogenesis and reverse the tumor immune suppressive microenvironment in both B16-bearing and 4T1-bearing mice.
Conclusions In this study, iNDV $3 \alpha-L P$ had several functions, such as tumor and vessel lysis, MIP- $3 \alpha$ immunotherapy, and binding to $\alpha v \beta 3$-expressing tumor and its neovasculature. iNDV3 $\alpha$-LP treatment significantly suppressed tumor angiogenesis and reversed the tumor immunosuppressive microenvironment. These findings offer a strong rationale for further clinical investigation into a combination strategy for oncolytic immunotherapy, such as the formulation iNDV3 $\alpha-L P$ in this study.

\section{INTRODUCTION}

Biotherapy with oncolytic viruses (OVs) is a promising method for treating cancer. ${ }^{12}$ OVs can infect and replicate in tumor cells and continue to infect others, leading to direct lysis of tumor cells. ${ }^{3-5}$ During this process, the infected tumor cells release many tumorassociated antigens, pathogen-associated molecular patterns, and damage-associated molecular patterns (DAMPS). These molecules can further stimulate adaptive immune responses against tumors, such as tumor immunogenic cell death (ICD), an immune process that can shift tumors from cold (immune desert) to hot (inflamed) tumors. ${ }^{6-8}$ However, OV monotherapy is unable to completely cure tumors, especially those that are deep and/or metastasized. Therefore, OVs require a combination of additional therapies to enhance their antitumor effects. This combination strategy has the added advantage of being able to deliver therapeutic transgenes to enhance antitumor activity through modulation of the host immune response. For example, oncolytic herpes virus (talimogene laherparepvec (T-VEC)) expressing granulocyte-macrophage colony-stimulating factor (GM-CSF) has been approved for the 
clinical treatment of non-resectable melanoma by the US Food and Drug Administration and the European Union. ${ }^{9-13}$ Intratumor administration of T-VEC led to a reduction in tumor size, but the clearance of the tumor remained incomplete. Case studies show that combining T-VEC with immune-checkpoint inhibitory monoclonal antibodies improves the antitumor effects. ${ }^{9-11}$ These clinical studies indicate that the combination of OVs with another immunotherapy strategy has additive or synergistic antitumor activities.

Angiogenesis, a process by which new blood vessels are formed or developed from existing blood vessels, plays an important role in tumor growth and metastasis. ${ }^{14}$ Antiangiogenesis therapies have been deeply investigated in various diseases, such as cancer and rheumatoid arthritis, for several decades, and they remain a research hotspot in this field. ${ }^{14-16}$ Various molecules, especially those that are overexpressed or specifically expressed on tumor neovasculature, have become the focus of therapeutic targets. Integrin alpha (v) beta (3) ( $\alpha v \beta 3)$ receptor is an integrin family member that is overexpressed on both tumor neovasculature and some tumor cells. ${ }^{17}$ Several studies have investigated the use of an $\alpha v \beta 3$ receptor conjugated to various nanoformulations, such as liposomes, for targeted therapy of cancers in murine models. ${ }^{18-20}$

iRGD is a cyclic nanopeptide with an amino acid sequence of CRGDKGPDC. iRGD has high tumortargeting efficiency and tumor-penetrating abilities if it is chemically conjugated to nanoformulations or to other therapeutic agents. ${ }^{21-23}$ Additionally, iRGD has a specific capability of binding to the integrin $\alpha v \beta 3$ receptor, which is highly expressed on tumor neovasculature but rarely on normal blood vessels. ${ }^{18-20}$ In a previous study, we engineered a recombinant Newcastle disease virus (NDV) that effectively expressed bioactive macrophage inflammatory protein-3 $\alpha$ (MIP-3 $\alpha$ ), a specific dendritic cell (DC) chemokine, and promoted tumor-specific antitumor immunity and reversal of the suppressive tumor microenvironment; this NDV showed optimal suppression of tumor growth in several murine models. ${ }^{24}$ Thus, in the current study, we encapsulated recombinant MIP-3 $\alpha$ NDV in an iRGD-liposome (iNDV3 $\alpha$-LP) to investigate its antitumor activities. Interestingly, we found that iNDV3 $\alpha$-LP treatment induced significant lysis of both tumor and endothelial cells and significantly promoted antitumor immunity. iNDV3 $\alpha$-LP treatment also significantly inhibited tumor neovasculature (the generation of new tumor vessels) and reversed the tumor suppressive microenvironment, resulting in a perfect inhibition of tumor growth in murine models.

\section{MATERIALS AND METHODS}

\section{Virus and detection of its replication in vitro}

The lentogenic NDV LaSota strain and its recombinant counterpart expressing MIP-3 $\alpha$ (NDV3 $\alpha$ ) previously made by our group were used in this study. ${ }^{24}$ The NDV and NDV3 $\alpha$ were cultured in embryonated chicken eggs. After serial dilution, the virus titers in A549 adenocarcinomic human alveolar basal epithelial cells were detected by immunofluorescence in a microplate system (ELX808IU, Bio-Tek, Winooski, Vermont, USA). Viral replication kinetics were detected in the following steps: $5 \times 10^{4} \mathrm{~B} 16$ murine melanoma, $4 \mathrm{~T} 1$ murine breast cancer cells or human umbilical vein endothelial cells (HUVECs) were infected with the NDV virus $\left(1 \times 10^{3} \mathrm{pfu}\right)$ in six-well plates at $0.05,0.5,1.0$, and 2.0 multiplicity of infection (MOI) in $100 \mu \mathrm{L}$. The culture medium was substituted with $1 \mathrm{~mL}$ of fresh medium, and the cells were continually cultured at $37^{\circ} \mathrm{C}$ in $1 \mathrm{~mL}$ Dulbecco's modified Eagle's medium or Roswell Park Memorial Institute (RPMI) 1640 medium supplemented with $250 \mathrm{ng} / \mathrm{mL}$ tosyl phenylalanyl chloromethyl ketone trypsin. Next, the virus replication at $24,48,72$, and 96 hours was detected as previously reported. ${ }^{2425}$

\section{Preparation and characterization of iNDV $3 \alpha-\mathrm{LP}$ and the control liposomes}

The iRGD-liposome loaded with NDV expressing MIP-3 $\alpha$ (iNDV3 $\alpha-\mathrm{LP}$ ), liposome loaded with NDV expressing MIP-3 $\alpha$ (NDV3 $\alpha-L P$ ), liposome loaded with single NDV (NDV-LP), and empty liposome (LP) were prepared as previously reported. ${ }^{20}{ }^{26}$ DSPE-PEG $_{2000}$-iRGD was first prepared through a reaction between iRGD and DSPE$\mathrm{PEG}_{2000}$-maleimide. In brief, iRGD and DSPE-PEG ${ }_{2000^{-}}$ maleimide were mixed at a 1.5:1 molar ratio in ultrapure water, followed by gentle stirring in a $4^{\circ} \mathrm{C}$ environment for 24 hours under nitrogen gas. To remove the unreacted impurities, the mixture was dialyzed in a dialysis bag (MWCO: 3500) for 48 hours in ultrapure water. The final solution in the dialysis bag was lyophilized for 48 hours and stored at $-20^{\circ} \mathrm{C}$ until required. To prepare the iNDV3 $\alpha-\mathrm{LP}$, DSPE-PEG $_{2000}$-iRGD, DSPE-PEG ${ }_{2000}$, cholesterol, and dipalmitoylphosphatidylcholine were mixed in chloroformin at 1:4:40:55 molecular ratios and then volatilized to remove organic solvents under nitrogen flow until a lipid film was generated. The lipid film was further hydrated at $37^{\circ} \mathrm{C}$ with $1 \times 10^{8} \mathrm{pfu} / \mathrm{mL}$ NDV3 $\alpha$ in $0.1 \mathrm{M}$ phosphate buffered saline (PBS; $\mathrm{pH} 7.4$ ) and vortexed for $5 \mathrm{~min}$ at room temperature. The resultant suspension was sequentially passed through a polycarbonate membrane of $200 \mathrm{~nm}$ pore size (Millipore, Bedford, Massachusetts, USA), and a Cellufine sulfate column was used to separate out the unconjugated NDVs. The control liposomes (LP, NDV-LP, and NDV3 $\alpha-$ LP) were prepared using the same method without the addition of corresponding ingredients. The size, average grain diameter, and zeta potential of iNDV3 $\alpha$-LP and the control liposomes were tested using a laser-based analyzer (Zeta sizer Nano ZS90, Malvern, UK). The morphological characteristics of the liposomes were detected by transmission electron microscopy (TEM). The encapsulation efficiency (EE) of NDV or NDV3 $\alpha$ was calculated using a previously reported formula. ${ }^{18} 20$

\section{Cell lysis and cytotoxicity assays}

The lactate dehydrogenase activity in the supernatant of B16, 4T1, and HUVECs was determined to evaluate 
the capability of the NDV or splenic monocytes to lyse tumor cells or HUVECs using a CytoTox 96 kit (Promega, Madison, Wisconsin, USA) as described previously. ${ }^{24} 25$ In brief, B16 cells, 4T1 cells, or HUVECs were infected with NDV virus $\left(1 \times 10^{3} \mathrm{pfu}\right)$ in six-well plates at $1 \mathrm{MOI}$ in $100 \mu \mathrm{L}$. After infection, $50 \mu \mathrm{L}$ aliquots were collected at 24, 48, 72, and 96 hours and transferred to another 96-well plate. After incubation for $60 \mathrm{~min}$ in the dark at room temperature, stop solution $(50 \mu \mathrm{L})$ was added to each well, and the optical density (OD) values were detected at $492 \mathrm{~nm}$ by an ELX808IU microplate system (Bio-Tek). To detect the cytotoxic T lymphocytes (CTLs), tumor cells (target) in logarithmic growth were mixed with splenic lymphocytes (effectors) in a series of $\mathrm{E}$ (effectors): T (target) ratios (5-40:1) and then plated at a total volume of $100 \mu \mathrm{L}$ in 96 -well plates. The plates were subsequently incubated in a $5 \% \mathrm{CO}_{2}$ atmosphere at $37^{\circ} \mathrm{C}$ for 8 hours. Following incubation, the $50 \mu \mathrm{L}$ aliquots were transferred to another 96-well plate, incubated and detected for OD values. The target cell lysis was expressed as a percentage at each E:T ratio as described previously. ${ }^{27}$

\section{Western blotting analysis}

MIP-3 $\alpha$, high mobility group box 1 protein (HMGB1), integrin $\alpha v \beta 3$ receptor, and sera IgG were analyzed by western blotting as described previously. ${ }^{24}$ The cells were infected with or without iNDV3 $\alpha$-LP, NDV3 $\alpha$-LP, NDV-LP, or LP at various MOIs for 24 hours, before collecting the cultured supernatants or cell lysates. Additionally, the sera from mice treated with different formulations were also collected. The cell lysates, sera, or supernatants were subjected to electrophoresis on 8\%-10\% SDS-PAGE gels. The proteins on the gels were transferred onto a polyvinylidene difluoride (PVDF) membrane (Bio-Rad). Next, the membranes were blocked with $10 \%$ non-fat milk at $4^{\circ} \mathrm{C}$ for 1 hour, washed with PBS three times, and then incubated with monoclonal antibodies (Abcam) against MIP-3 $\alpha$, HMGB1, $\alpha v \beta 3$ receptor, or IgG at a 1:200 dilution. Eventually, the PVDF membranes were stained with enhanced chemiluminescence (ECL) substrates for $30 \mathrm{~min}$, and the resultant pictures were obtained using an ECL system (Amersham Biosciences, UK).

\section{Isolation of single cells}

Murine bone marrow derived dendritic cells (BMDCs), spleen lymphocytes and tumor-infiltrating lymphocytes (TILs) were isolated for analysis. BMDCs were isolated from 8 to 10 week-old female BALB/c or C57BL/6 mice as previously described. ${ }^{28}$ Following depletion of the erythrocytes, the isolated bone marrow cells $\left(1 \times 10^{5} /\right.$ well) were cultured in RPMI-1640 (HyClone, Thermo Scientific, Waltham, Massachusetts, USA) supplemented with GM-CSF $(100 \mathrm{U} / \mathrm{mL})$ and IL-4 $(20 \mathrm{ng} / \mathrm{mL})$. The non-adherent cells were depleted on days 2 and 4, while the adherent cells were transferred to another six-well plate on day 6 . The cells were incubated for a further 6 days, and the resultant non-adherent cells with typical morphological characteristics of BMDCs were collected for subsequent experiments. To isolate the spleen lymphocytes, spleens were minced into small pieces with a scissor and then pressed through $100 \mu \mathrm{m}$ cell strainers (BD Biosciences, Franklin Lakes, New Jersey, USA). The erythrocytes in the cell suspensions were further lysed with ACK buffer and then filtered through a cell strainer $(100 \mu \mathrm{m})$. The resultant cell suspensions were used for subsequent experiments. To isolate single tumor cells, B16 and 4T1 tumor tissues were minced with a TissueLyser II instrument (Qiagen, Germany) and digested in culture medium with $0.8 \mathrm{mg} / \mathrm{mL}$ Dispase II, $0.2 \mathrm{mg}$ / $\mathrm{mL}$ collagenase $\mathrm{P}$, and $0.1 \mathrm{mg} / \mathrm{mL}$ DNase I (all from BD Biosciences, San Jose, California, USA) for $30 \mathrm{~min}$ at $37^{\circ} \mathrm{C}$. The isolated cells from B16 and $4 \mathrm{~T} 1$ tumors were filtered through a cell strainer $(70 \mu \mathrm{m})$ and purified on a Ficoll gradient as previously described. ${ }^{24}$

\section{In vitro DC chemotaxis assay}

The in vitro DC chemotaxis assay was performed in a 24-well Transwell chamber kit (Corning Costar, New York, USA) as previously reported. ${ }^{24}{ }^{27}$ In brief, $5 \times 10^{4}$ DCs dissolved in $200 \mu \mathrm{L}$ bovine serum albumin medium $(5 \%)$ were plated in the upper chamber, while indicated percentages of supernatants from iNDV3 $\alpha$-LP-treated, NDV3 $\alpha$-LP-treated, NDV-LP-treated, or LP-treated tumor or HUVECs were added to a volume of $750 \mu \mathrm{L}$ in the bottom chamber. The Transwell chambers were placed in an incubator supplemented with $5 \% \mathrm{CO}_{2}$ at $37^{\circ} \mathrm{C}$ for 3 hours. Subsequently, the filter between the upper and bottom chamber was removed and washed with Hanks' Balanced Salt Solution, fixed, and stained on a slide according to the Transwell chamber kit protocol. The number of chemotactic DCs on the bottom chamber was counted using a microscope (80i, NIKON, Japan) at 200× magnification.

\section{Flow cytometry (FCM) analysis}

FCM analysis was performed to analyze the expression of marker molecules, including CD11c, DEC205, CD4, CD8, CD45, CD25, Foxp3, and Gr-1, on the cell membranes, as well as intracellular cytokines, including IFN- $\gamma, \mathrm{TNF}-\alpha$, and IL-2. ${ }^{24}$ Cells stained with fluorescent dyes, such as CFSE and DiI, were also analyzed by FCM. The cells isolated from various tissues were incubated with the corresponding fluorescent dyes or monoclonal antibodies conjugated to FITC, PE, PerCP-Cy5.5, or APC for $30 \mathrm{~min}$ at $4^{\circ} \mathrm{C}$ in 1:100-150 dilutions. The following monoclonal antibodies were purchased from eBioscience (San Diego, California, USA) or BD Biosciences: anti-DEC205, antiCD11c, anti-CD45, anticalreticulin (anti-CRT), anti-CD8, anti-IFN- $\gamma$, anti-TNF- $\alpha$, anti-CD4, anti-CD25, anti-FOXP3, anti-Gr-1, anti-CD206, and anti-F4/80. Before intracellular cytokine staining, $2 \times 10^{6}$ splenocytes were stimulated with tumor lysates $(5 \mu \mathrm{g} / \mathrm{mL})$ in culture medium with $10 \%$ fetal calf serum and $2 \mu \mathrm{g} / \mathrm{mL}$ brefeldin A (BD Bioscience) for 6 hours at $37^{\circ} \mathrm{C}$. The intracellular cytokines in splenocytes or TILs were stained using the Cytofix/Cytoperm kit (BD Bioscience) as per the manufacturer's protocol. 
The stained cells were detected by FCM (CyFlow Cube 6; Sysmex, Japan), and the resultant data were analyzed to capture the FCM images with FlowJo software version 10 (Tree Star, Ashland, Oregon, USA).

\section{Fluorescent observation of cell morphology}

Fluorescent staining with dyes or monoclonal antibodies was performed to observe whether CRT was present on the cell surface and whether the fluorescence-labeled liposome iNDV $3 \alpha-L P$ targets the $\alpha v \beta 3$ receptor in vitro and the blood vessels in vivo, as previously reported. ${ }^{151628}$ For in vitro observation, B16, 4T1, or HUVECs $\left(1 \times 10^{5} /\right.$ well) were incubated in six-well plates and infected with NDV-encapsulated or NDV3 $\alpha$-encapsulated liposome formulations with equivalent 1 MOI NDV or treated with empty LP. The cells were first incubated at $37^{\circ} \mathrm{C}$ in a $5 \% \mathrm{CO}_{2}$ atmosphere for $10 \mathrm{~min}$ and then washed thrice with PBS. After being fixed in $4 \%$ paraformaldehyde at room temperature for $30 \mathrm{~min}$, the cells were stained with anti-CRT or anti- $\alpha v \beta 3$ antibodies (BD Bioscience) in 1:250 dilutions at $4^{\circ} \mathrm{C}$ overnight and then washed three times with cold PBS. Following washing, the cells were stained with a FITC-labeled secondary antibody at 1:300 dilutions for $60 \mathrm{~min}$ at room temperature. Additionally, the cell membrane and nuclei were stained with DiI (MedChemExpress, USA) and DAPI (Beyotime Biotechnology, China) for $15 \mathrm{~min}$. Images were captured by a confocal microscope (FV1000, Olympus, Japan). To observe whether iNDV3 $\alpha$-LP targets blood vessels in vivo, B16- or 4T1-bearing mice were randomly grouped and intravenously injected with DiI-conjugated iNDV3 $\alpha$-LP, NDV3 $\alpha$-LP, NDV-LP, or LP. Thirty minutes after injection, $10 \mathrm{mg} / \mathrm{mL}$ fluorescein isothiocyanate conjugate (FITC)-Dextran (Sigma, USA) was intravenously injected into the mice by tail vein. Ninety minutes after FITCdextran injection, the mice were sacrificed, and the tumors were removed into cryo-embedding compound OCT (Tissue-Tek, Sacura) for frozen sectioning, and the images were captured by a confocal fluorescence microscope (FV1000, Olympus, Japan).

\section{Mice tumor models and liposome treatment}

The syngeneic C57BL/6 or BALB/c mice (6-8 weeks old) were chosen for the establishment of B16 or $4 \mathrm{~T} 1$ tumor models. In brief, B16 or $4 \mathrm{~T} 1$ cells $\left(5 \times 10^{5}\right.$ in $100 \mu \mathrm{L}$ PBS) were subcutaneously implanted in the right flank. The mice were randomly divided into four groups ( $\mathrm{n}=10$ per group) based on receiving tail vein injection of iNDV3 $\alpha$-LP, NDV3 $\alpha$-LP, NDV-LP ((NDV or NDV3 $\alpha$ $2 \times 10^{7} \mathrm{pfu}$ ), or LP in $100 \mu \mathrm{L}$ PBS. The injections were performed on day 3 after tumor cells were implanted as previously reported..$^{25}$ The treatment was repeated once a week for a total of four treatments. The tumor volumes and images were taken in 3-day intervals by a handheld tumor-detecting instrument (TM900, Peira Scientific Instruments, Belgium). At the same time, the mouse survival was recorded.

\section{Calculation of synergistic indexes (SIs)}

In this study, we calculated an SI using the tumor volume, tumor angiogenesis, and TILs to evaluate whether the iNDV3 $\alpha$-LP treatment induced synergistic antitumor activities through synergistic inhibition of neovasculature formation and reversal of the tumor suppressive microenvironment as described previously. ${ }^{15}$ The mean values of these parameters were used to calculate the SI for each group. In brief, an observed relative ratio (ORR) of the NDV-LP, NDV3 $\alpha$-LP, and iNDV3 $\alpha$-LP groups was obtained by dividing the mean of each group by that of the LP group. The expected relative ratio (ERR) of the iNDV3 $\alpha$-LP group was obtained by multiplying the ORR of the NDV-LP group and that of the NDV3 $\alpha-L P$ group. The SI of iNDV3 $\alpha$-LP treatment was calculated using the formula: $\mathrm{SI}=\mathrm{ERR} / \mathrm{ORR}(\mathrm{ORR}<1)$ or $\mathrm{ORR} / \mathrm{ERR}$ $(\mathrm{ORR}>1)$, where $\mathrm{SI}>1$ indicates a synergistic effect.

\section{ELISA and enzyme-linked immunospot (ELISPOT) assay}

The concentrations of HMGB1 and ATP in the supernatants of tumor cells treated with iNDV3 $\alpha-\mathrm{LP}$, control, and IgG antibody and its subtypes in pooled sera from the liposome-treated mice were quantified by commercial ELISA kits (Wuhan Boster Biological Technology, China) according to the manufacturer's protocol. Additionally, ELISPOT was performed to enumerate the number of splenocytes that produced IgG antibodies specific to B16 or $4 \mathrm{~T} 1$ tumors as previously reported. ${ }^{27}$ In brief, tumor lysate $(30 \mu \mathrm{g} / \mathrm{mL})$ was first added to PVDF-bottomed 96-well plates (Millipore, Billerica, Massachusetts, USA). Next, the various splenocytes isolated from the spleen of the mice treated with iNDV3 $\alpha$-LP, NDV3 $\alpha$-LP, NDV-LP, or $\mathrm{LP}$ were incubated in the plates at $37^{\circ} \mathrm{C}$ in a $5 \% \mathrm{CO}_{2}$ atmosphere for 6 hours. The plate was then washed thrice with PBS and stained with ECL substrates using the protocol outlined for western blotting. The number of spots on the PVDF membrane was considered to indicate the number of IgG-secreted cells.

\section{Immunohistochemistry}

Immunohistochemistry was performed to detect the microvessels in B16 and 4T1 tumor sections as described previously. ${ }^{24}$ In brief, frozen sections $(5 \mu \mathrm{m})$ from tumors treated with iNDV3 $\alpha$-LP, NDV3 $\alpha-L P$, NDV-LP, or LP were placed on slides, fixed with acetone for $20 \mathrm{~min}$, and airdried for $60 \mathrm{~min}$. Next, the slides were washed three times with PBS and then incubated in PBS containing $5 \%$ normal goat serum for $90 \mathrm{~min}$ at room temperature. The slides were further incubated overnight at $4^{\circ} \mathrm{C}$ in PBS with rat antimouse antibodies against CD41 (BD Bioscience) at a 1:50 dilution. Subsequently, the sections were further stained with streptavidin biotin-labeled reagents (Dako LSAB kit, peroxidase; Dako), and the images were obtained under a microscope (80i, NIKON, Japan).

\section{Alginate encapsulation assay}

An alginate encapsulated tumor cell assay to quantify tumor microvessels was performed as described previously. ${ }^{15} 16$ 
In brief, B16 or $4 \mathrm{~T} 1$ cells $\left(1 \times 10^{6}\right)$ in a solution of $1.5 \%$ sodium alginate (Sigma, St. Louis) were added dropwise to a swirling calcium chloride solution $(250 \mathrm{mM})$. During this process, alginate beads containing approximately $1 \times 10^{4}$ tumor cells in each bead were formed. Four beads for each mouse were implanted subcutaneously into an incision in the dorsal side. On day 14, the mice were injected with $10 \mathrm{mg} / \mathrm{kg}$ FITC-dextran in $100 \mu \mathrm{L}$ PBS via the tail vein (Sigma). The alginate beads were surgically removed and squashed in a TissueLyser II (Qiagen). The fluorescence intensity of the beads was quantified against a standard curve of FITC-dextran by a microplate reader (ELX808IU, Bio-Tek).

\section{Statistical analysis}

The data are presented as the mean $\pm \mathrm{SD}$, and all data were analyzed by GraphPad-Prism Software (San Diego, California USA). One-way or two-way analysis of variance (ANOVA), followed by a Tukey's multiple comparisons test, was performed to compare the differences among the experimental groups. Animal survival was graphed as Kaplan-Meier survival curves and analyzed with the logrank (Mantel-Cox) test.

\section{RESULTS}

\section{Characterization of engineered liposomes}

The procedure for the preparation of iRGD-liposome loaded with NDV expressing MIP-3 $\alpha$ (iNDV3 $\alpha$-LP) is illustrated in figure 1A. Other liposomes used for controls, such as empty LP, NDV-LP or NDV3 $\alpha$-LP, were also prepared by following a similar procedure but without corresponding ingredients. Successful preparation of the four liposomes was confirmed by their typical morphology visualized by TEM (figure 1B). The diameter of iNDV3 $\alpha$-LP was slightly larger than that of LP and NDV-LP but almost equal to that of NDV3 $\alpha$-LP (figure 1C,D). The diameters, zeta potential, and polydispersity are shown in figure 1D, showing no significant difference among the four liposomes. Additionally, the EE of NDV and NDV3 $\alpha$ was similar (approximately $70 \%$ ) in NDV-LP, NDV3 $\alpha-L P$, and iNDV3 $\alpha-L P$ (figure 1D). These data indicate that LP, NDV-LP, NDV3 $\alpha-L P$, and iNDV3 $\alpha$-LP are suitable for use in the study.

\section{Recombinant MIP-3 $\alpha$ NDVs in iRGD-liposomes increase the oncolytic activities of $\alpha v \beta 3$-expressing cells and express similar bioactivities of MIP-3 $\alpha$}

Previous studies have shown that $4 \mathrm{~T} 1$ breast cancer cells and vascular endothelial cells express $\alpha v \beta 3^{20}$ and that NDV proliferation is the most efficient in B16 tumor cells. $^{24}{ }^{25}$ Thus, we first observed whether the NDVs encapsulated in iNDV3 $\alpha-L P$, NDV $3 \alpha-L P$, and NDV-LP retained their oncolytic ability and replicative capacity in 4T1 and B16 tumor cells and HUVECs. The cells treated with equivalent titers of NDV or NDV3 $\alpha$ in iNDV3 $\alpha-L P$, NDV3 $\alpha-L P$, and NDV-LP showed similar levels of viral replication (figure 2A) and cell lysis (figure 2B) as the wild-type NDV in B16 cells. However, the level of viral replication and cell lysis in both $4 \mathrm{~T} 1$ and HUVECs was significantly increased, especially when the MOI was low
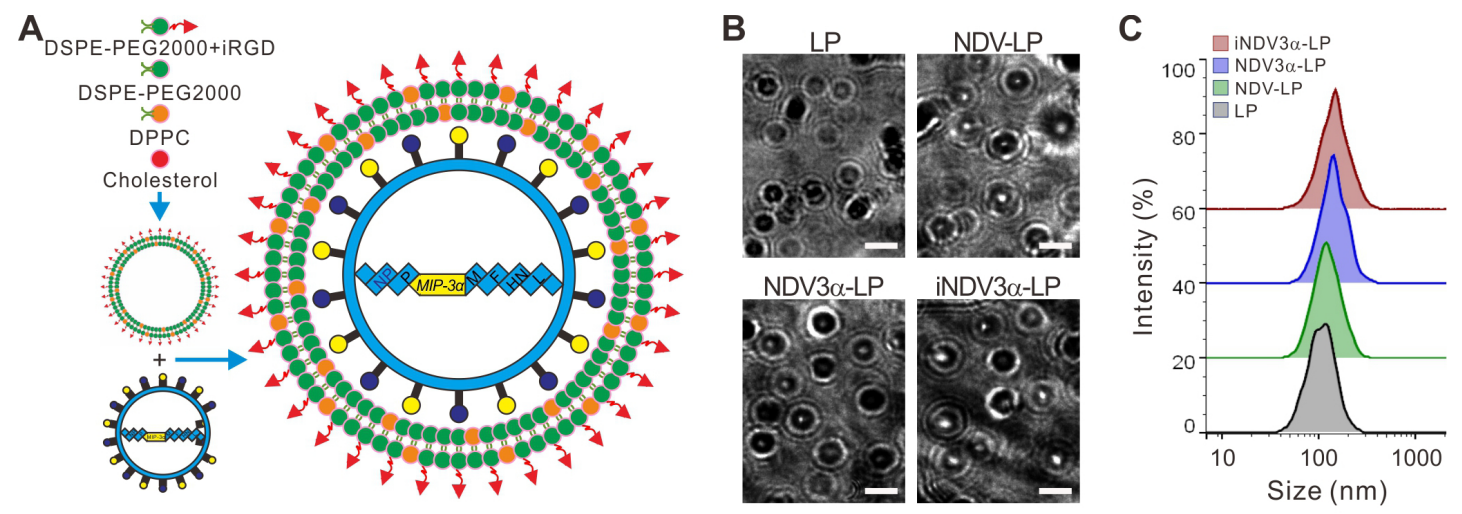

\begin{tabular}{|c|c|c|c|c|}
\hline Liposome & Diameter (nm) & Zeta potential $(\mathrm{mV})$ & Polydispersity (PDI) & EE $(\%)$ \\
\hline iNDV3a-LP & $149.47 \pm 52.44$ & $-37.66 \pm 8.35$ & $0.169 \pm 0.002$ & $70.66 \pm 6.496$ \\
\hline NDV3a-LP & $145.38 \pm 45.92$ & $-35.45 \pm 7.45$ & $0.183 \pm 0.006$ & $64.65 \pm 5.499$ \\
\hline NDV-LP & 126. $27 \pm 42.26$ & $-34.71 \pm 10.34$ & $0.188 \pm 0.007$ & $66.61 \pm 4.375$ \\
\hline LP & $109.39 \pm 34.83$ & $-22.27 \pm 9.25$ & $0.185 \pm 0.005$ & \\
\hline
\end{tabular}

Figure 1 Construction and characteristic evaluation of the liposome formulation NDV-MIP3 $\alpha$. (A) Schematic presentation of the construction of iNDV3 $\alpha$-LP and three control liposomes NDV3 $\alpha$-LP, NDV-LP and Lp. (B) TEM images of liposomes LP, NDV-LP, NDV3 $\alpha$-LP, and iNDV3 $\alpha$-LP. (C) Size distribution of LP, NDV-LP, NDV3 $\alpha$-LP, and iNDV3 $\alpha$-LP. (D) Basic characteristics of LP, NDV-LP, NDV3 $\alpha$-LP, and iNDV3 $\alpha$-LP. LP, empty liposome; MIP-3 $\alpha$, macrophage inflammatory protein-3 $\alpha$; NDV; Newcastle disease virus; NDV-LP, liposome encapsulated with wild-type LP NDV; NDV3 $\alpha$-LP, liposome encapsulated with the recombinant MIP-3 $\alpha$ NDV; iNDV3 $\alpha$-LP, iRGD-directed liposome encapsulated with the recombinant MIP-3 $\alpha$ NDV. 

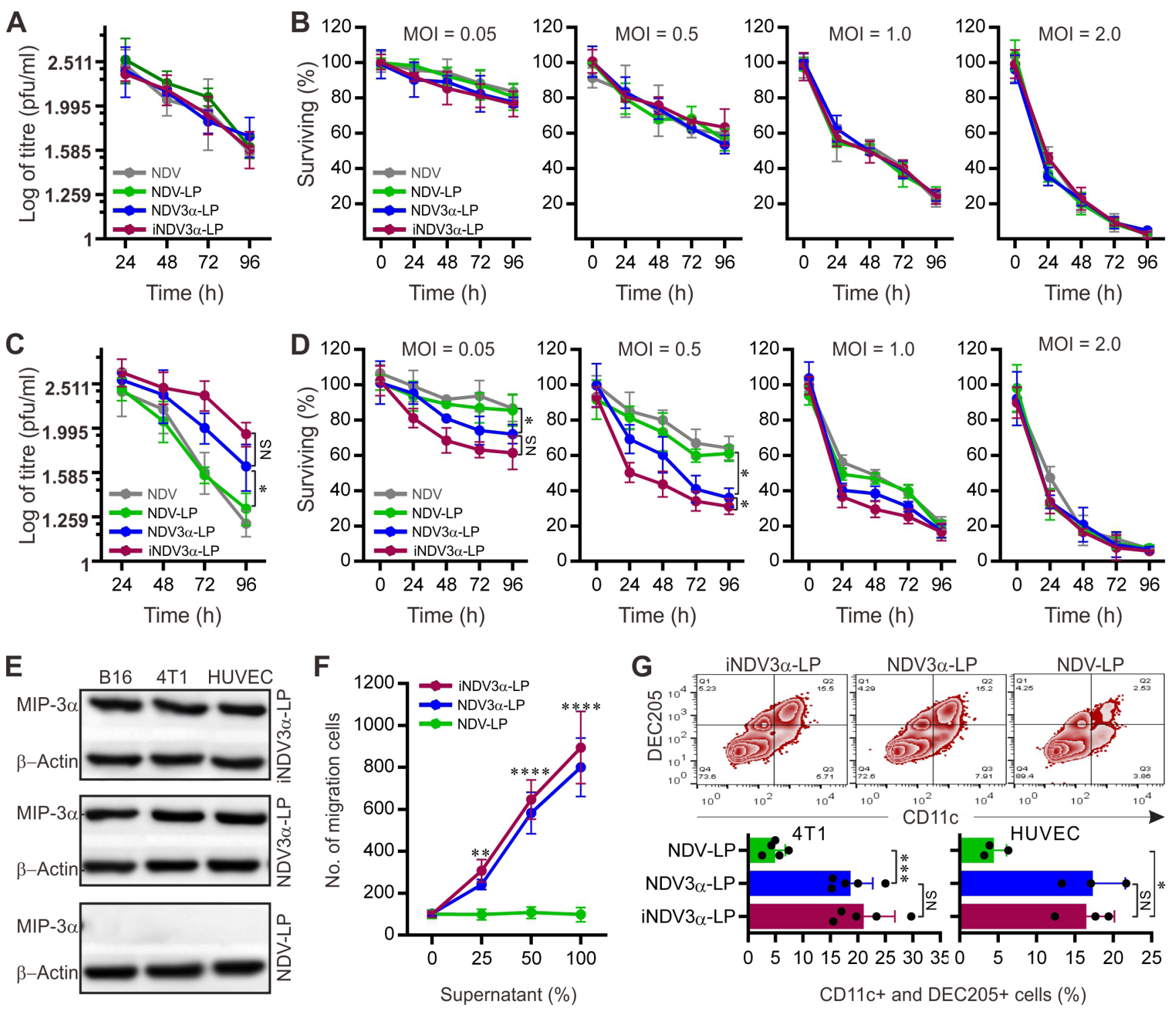

Figure 2 Evaluation on replication and tumor-lysis capabilities of the NDVs and MIP-3 $\alpha$ activity in the liposomes. (A and C) vVrus replication of $1 \mathrm{MOI} N \mathrm{NV}$ or NDV3 $\alpha$ at the indicated time points in B16 (A) and 4T1 (B) cells. (B and D) Lysis of B16 (B) or 4T1 (D) cells by indicated formulations at the indicated MOI and time points. (E) Expression of MIP-3 $\alpha$ in B16 and 4T1 cells treated with indicated formulations. (F) In vitro chemotaxis of DCs induced by the supernatants from B16 cells infected with $1 \mathrm{MOI} N D V$ or NDV3 $\alpha$ in the indicated formulations. (G) In vivo chemotaxis for DCs in mouse abdomen injected with supernatants from B16 or 4 T1 cells treated with the indicated formulations. Data are plotted as mean \pm SD; two-way ANOVA with Tukey multiple comparisons: ${ }^{*} \mathrm{p}<0.05,{ }^{* *} \mathrm{p}<0.01,{ }^{* *} \mathrm{p}<0.001,{ }^{* \star * *} \mathrm{p}<0.0001$. ANOVA, analysis of variance; DCs dendritic cells; $\mathrm{MOI}$, multiplicity of infection; NDV $3 \alpha$, recombinant NDV encoding MIP-3 $\alpha$.

(figure 2C,D and online supplemental figure S1A,B). These data suggest that recombinant NDV3 $\alpha$ encapsulated in iNDV3 $\alpha$-LP or NDV3 $\alpha$-LP had similar bioactivities in B16 cells but induced more cell lysis in CT41 cells and HUVEC compared with wild-type NDV encapsulated in NDV-LP. In agreement with our previous study, ${ }^{24} 1 \mathrm{MOI}$ was a moderate titer of NDV or NDV3 $\alpha$ for tumor cell lysis (figure 2A-D and online supplemental figure S1A,B). Considering that robust cell lysis induced by NDVs was not necessary for the induction of antitumor immunity, we chose 1 MOI of NDV3 $\alpha$ and its wild-type counterpart (NDV) to perform the subsequent experiments in order to simplify our study and save resources.
Western blot analysis verified the expression of MIP-3 $\alpha$ in B16, 4T1, and HUVECs infected with iNDV3 $\alpha-L P$ and NDV3 $\alpha$-LP but not in the cells infected with NDV-LP (figure 2E). The bioactive MIP-3 $\alpha$ in the culture supernatants was confirmed by a DC chemotaxis assay in Transwell chambers and directly in an in vivo mouse model. ${ }^{24} 2728$ As predicted, the supernatants of $\mathrm{B} 16,4 \mathrm{~T} 1$, and HUVECs infected with iNDV3 $\alpha$-LP and NDV3 $\alpha$-LP showed significant chemotactic effects for DCs compared with the NDV-LP-infected cells (figure $2 \mathrm{~F}$ and online supplemental figure S1C,D). In the in vivo mouse model, the supernatants from B16, 4T1, and HUVECs infected with 
iNDV3 $\alpha-L P$, NDV3 $\alpha-L P$, or NDV-LP were injected into the abdominal cavities. On day 3 after supernatant injection, cells in the abdominal cavities were collected for FCM analysis. Compared with the NDV-LP group, more CD11c and DEC205 double-positive DCs were found in the iNDV3 $\alpha$-LP and NDV3 $\alpha-\mathrm{LP}$ groups (figure 2G and online supplemental figure S1E). Taken together, these data indicate that the MIP-3 $\alpha$ expressed in iNDV3 $\alpha$-LP and NDV3 $\alpha-\mathrm{LP}$ is functionally bioactive.

\section{iNDV3 $\alpha$-LP increased the production of DAMPs in tumor cells and HUVECS}

The production of DAMPs induced by OVs is considered a potential strategy for tumor immunotherapy. ${ }^{29} 30$ Thus, DAMP markers were detected in B16 and 4T1 tumor cells infected with iNDV3 $\alpha$-LP, NDV3 $\alpha$-LP, or NDV-LP at 1 MOI. Compared with cells infected with NDV-LP, the expression (c) and secretion (s) of HMGB1 were at least equivalent or even significantly higher in both B16 and 4T1 cells infected with iNDV3 $\alpha$-LP or NDV3 $\alpha$-LP (figure 3A). Additionally, sHMGB1 in the supernatant was detected by ELISA and demonstrated similar results as western blot analysis in B16 and 4T1 cells (figure 3B). Consistent with the HMGB1 expression, the release of ATP in the supernatants from the cells infected with iNDV3 $\alpha$-LP or NDV3 $\alpha$-LP was the same, or even increased, compared with that from the cells infected with NDV-LP (figure 3C). Additionally, CRT on the outer leaflet of the cell membrane (ecto-CRT) was directly observed by immunofluorescence staining and quantitatively analyzed by FCM at 24 hours after the B16 and $4 \mathrm{~T} 1$ cells were infected with iNDV3 $\alpha$-LP, NDV3 $\alpha$-LP, NDV-LP, or LP. Compared with the LP-treated cells, the results showed significant translocation of CRT onto the cell surface in both B16 and $4 \mathrm{~T} 1$ cells treated with iNDV3 $\alpha-L P$, NDV3 $\alpha$-LP, NDV-LP (figure 3D). Interestingly, the FCM results showed that MIP3 $\alpha$-expressing NDV liposomes promoted an increase in CRT translocation, which was more significant in $4 \mathrm{~T} 1$ cells (figure 3E). Taken together, these data demonstrate that both iNDV3 $\alpha$-LP and NDV3 $\alpha$-LP were superior in their ability to induce DAMP release in B16 and 4T1 cells, and the phenomenon was more significant in iNDV3 $\alpha-$ LP-infected cells.

\section{iNDV3 $\alpha$-LP targets iRGD-specific receptors on tumor cells and HUVECS}

Our previously mentioned results (figures 2 and 3 ) show that iNDV3 $\alpha$-LP induced more significant intensities of cell lysis and DAMP production in 4T1 cells than in B16 cells. We hypothesized that these phenomena are related to iRGD-specific receptors on the cells. Therefore, we detected whether $\alpha \mathrm{v} \beta 3$ was expressed on B16, 4T1, and HUVECs, by western blotting. As predicted, $\alpha v \beta 3$ expression was found in $4 \mathrm{~T} 1$ and HUVECs but not in B16 cells (figure 4A). To identify whether iNDV3 $\alpha$-LP specifically adhered to $\alpha v \beta 3$-expressing cells, iNDV3 $\alpha$-LP, NDV3 $\alpha$-LP, NDV-LP, and LP were labeled with FITC (green), the cell membrane was labeled with DiI (red), and the nucleus was stained with DAPI. We then observed the cells using confocal microscopy, before FCM analysis. Consistent with the western blotting results, colocalization of green and red signals (yellow) was found on 4T1 and HUVECs treated with iNDV3 $\alpha$-LP but not on B16 cells (figure 4B). As predicted, no colocalization of green and red signals was found in the three cell lines treated with NDV3 $\alpha-L P$, NDV-LP, or LP (online supplemental figure S1F). These findings were further confirmed by FCM analysis (figure 4E).

To detect whether iNDV3 $\alpha$-LP could directly target tumor blood vessels, B16 and 4T1 tumor models were established in syngeneic mice. After the tumor masses were palpable, FITC-conjugated dextran and DiI-labeled liposomes were injected into tumor-bearing mice, and 20 min later, the tumor masses were removed for frozen sectioning and then observed by fluorescence microscopy. The results showed that colocalization of green and red signals (yellow) was only observed in the sections of 4T1 tumors injected with DiI-labeled iNDV3 $\alpha$-LP but not in the sections of $4 \mathrm{~T} 1$ tumors injected with NDV3 $\alpha-\mathrm{LP}$, NDVLP, or LP (figure 4F). As predicted, no colocalization of green and red signals was found in any sections of B16 tumors (figure 4F). Taken together, these data strongly suggest that iNDV3 $\alpha$-LP targets iRGD-specific receptors on tumor cells and HUVECs.

\section{iNDV3 $\alpha$-LP induces antitumor effects in mouse tumor models}

The antitumor activities of the engineered liposomes iNDV3 $\alpha-L P$, NDV3 $\alpha-L P$, NDV-LP, and LP were evaluated in B16 and 4T1 tumor models. These liposomes were injected into the tail vein of the model mice when the tumor masses were palpable on day 5 after inoculation of tumor cells. Figure 5A shows the tumor masses in 4T1-bearing mice on day 25 after inoculation of tumor cells; the results show effective antitumor activities of NDV-LP, NDV3 $\alpha-L P$, and iNDV3 $\alpha-L P$, but the most effective activities were observed in mice treated with iNDV3 $\alpha$-LP. Treatment with NDV-LP, NDV3 $\alpha$-LP, or iNDV3 $\alpha$-LP suppressed tumor growth and increased survival rates in both $\mathrm{B} 16-$ and 4T1-bearing mice, but treatment with iNDV3 $\alpha$-LP was superior to other formulations (figure 5B,C).

The previous data (figures 2-4) indicate that iNDV3 $\alpha$-LP, but not NDV3 $\alpha$-LP, can directly lyse and bind to HUVECs in vitro and target blood vessels in vivo. The engineered liposome iNDV3 $\alpha$-LP used in this study contains several strategies, such as iRGD-directed HUVEC lysis, tumorvessel targeting, and immune enforcing by MIP-3 $\alpha$. Thus, treatment with the iNDV3 $\alpha$-LP liposome could be considered as a combination therapy, NDV3 $\alpha$-LP as NDV combined with MIP-3 $\alpha$ immunotherapy, NDV-LP as only NDV monotherapy, and LP as not treatment control. To investigate whether the combination of these additional strategies by iNDV3 $\alpha$-LP could enhance antitumor immunity, the mean tumor volume of both B16 and 4T1 cells at different time points was used to calculate the SI. The resultant SIs at most of the time points 

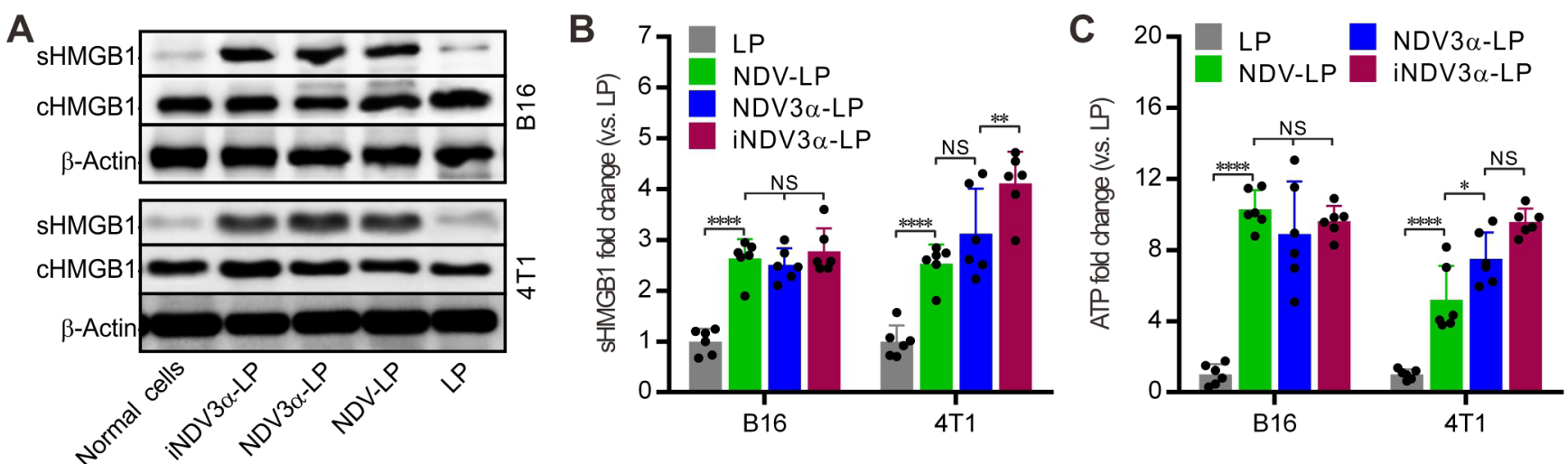

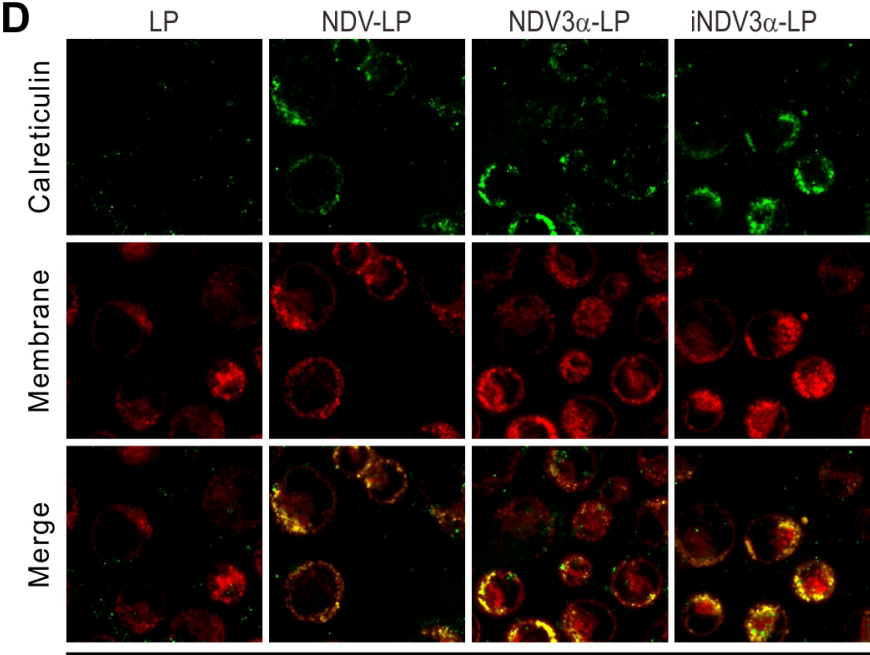

B16

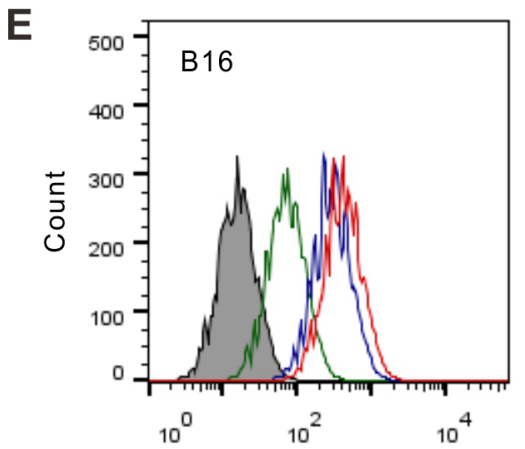

Calreticulin (fluorescence intensity)

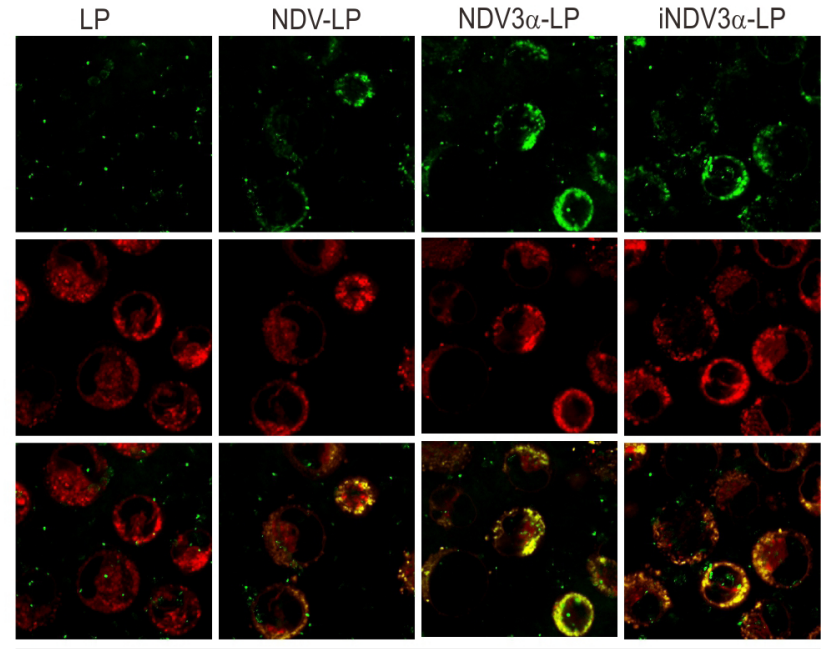

$4 \mathrm{~T} 1$

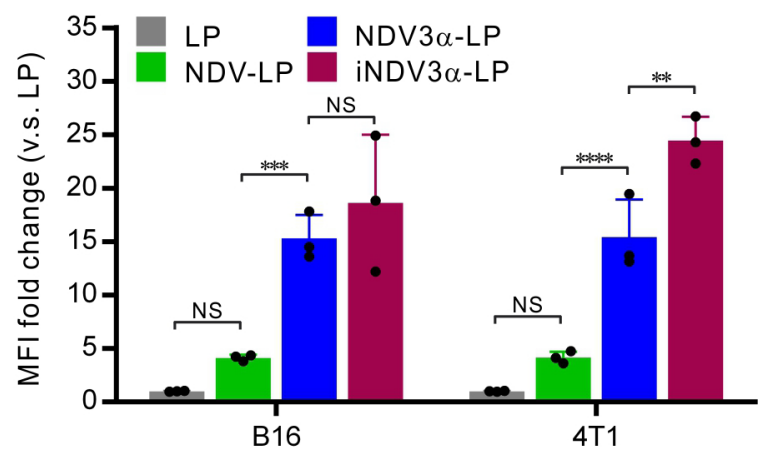

Figure 3 iNDV3 $\alpha$-LP treatment induces the immunogenic tumor cell death. (A) HMGB1 in B16 and 4T1 cell supernatants (sHMGB1) and whole-cells (cHMGB1) treated with the indicated formulations by western blot. (B) The titers of sHMGB1 in B16 and 4 T1 cell supernatants by ELISA. (C) ATP released in B16 or 4T1 cell supernatants by ELISA. (D) CRT translocation to B16 or $4 \mathrm{~T} 1$ cell surfaces by two counterstains with an FITG-conjugate anti-CRT antibody (green) and Dil (red). (E) FCM analysis of the CRT intensity on the B16 and 4T1 cell surface, showing the representative images and MFI fold change. CRT, calreticulin; FCM, flow cytometry; HMGB1, high mobility group box 1; iNDV3 $\alpha$-LP, iRGD-liposome loaded with NDV expressing MIP-3 $\alpha$; sHMGB1, secreted HMGB1.

were $>1$ (figure 5D), suggesting that the combination of the above-mentioned strategies of iNDV3 $\alpha$-LP induces stronger antitumor activities in both B16 and 4T1 models.

iNDV3 $\alpha$-LP induces tumor-specific cellular immunity and humoral immunity

Both cellular and humoral immunity are major arsenals against tumors. In the current study, tumor-specific
CTLs were detected using splenocytes as effector cells and 4T1 or B16 cells as target cells. Target cell lysis was significantly evidenced in the iNDV3 $\alpha$-LP, NDV3 $\alpha$-LP, and NDV-LP groups, but the greatest lysis was observed in the iNDV $3 \alpha-\mathrm{LP}$ group (figure $6 \mathrm{~A}, \mathrm{~B}$ ). In this study, an in vivo method was used to detect tumor-specific CTLs by injecting CFSE-conjugated 4T1 or B16 cells into the 
A
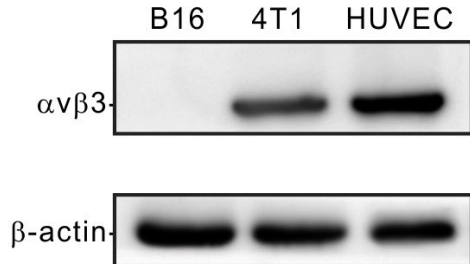

B

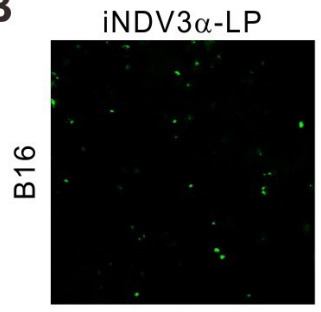

Membrane

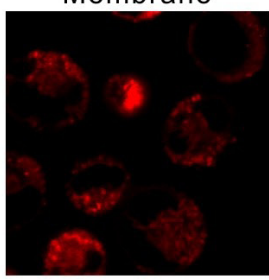

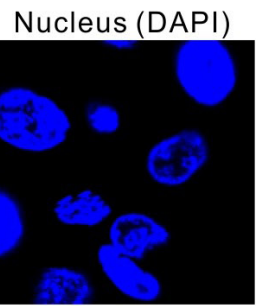

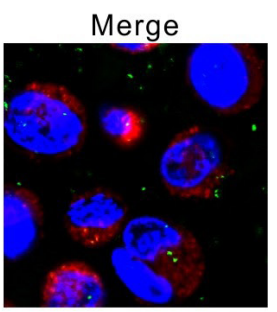

C
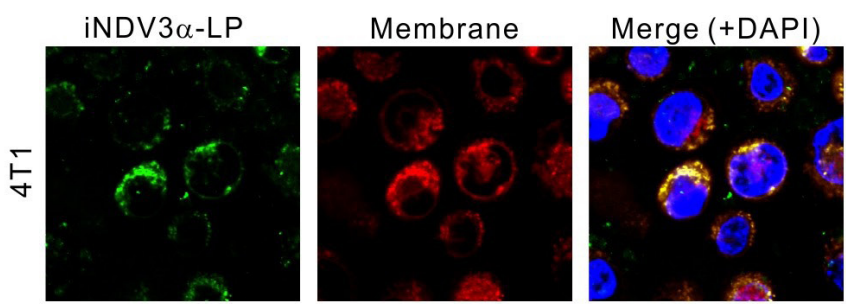

D
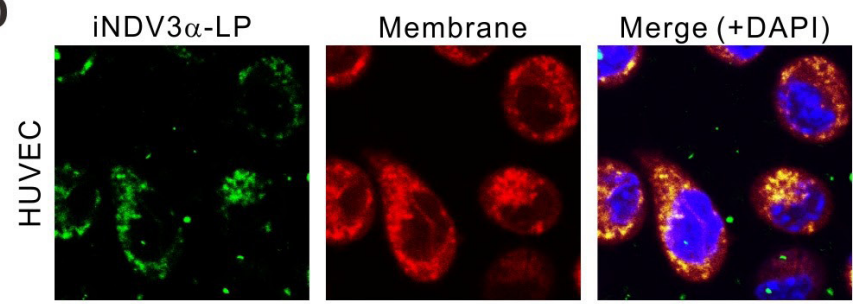

E
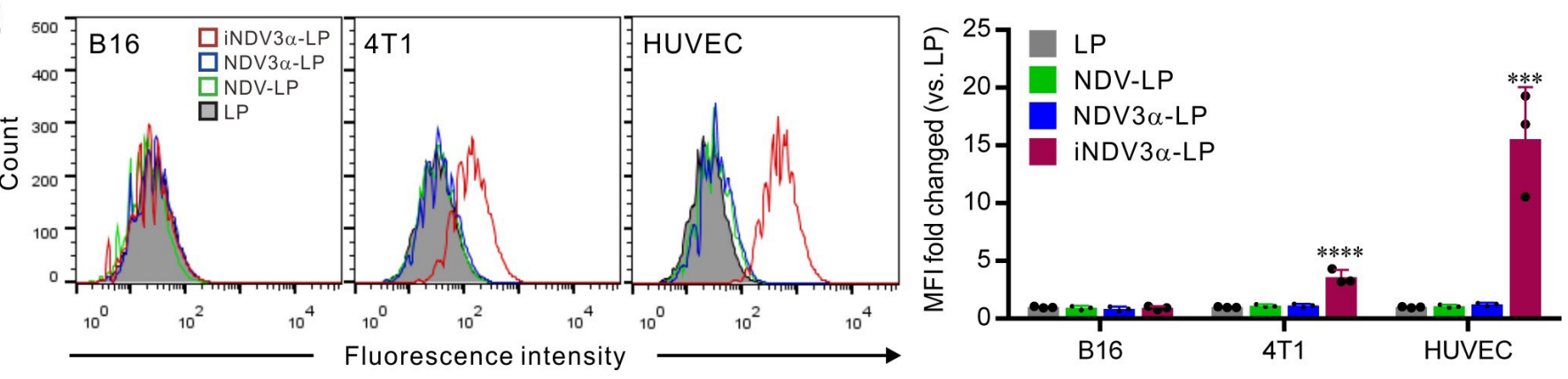

F LP
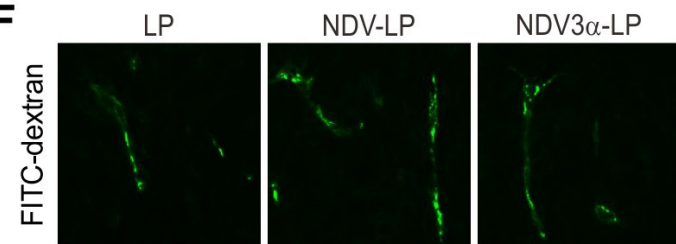

iNDV3 $\alpha-L P$
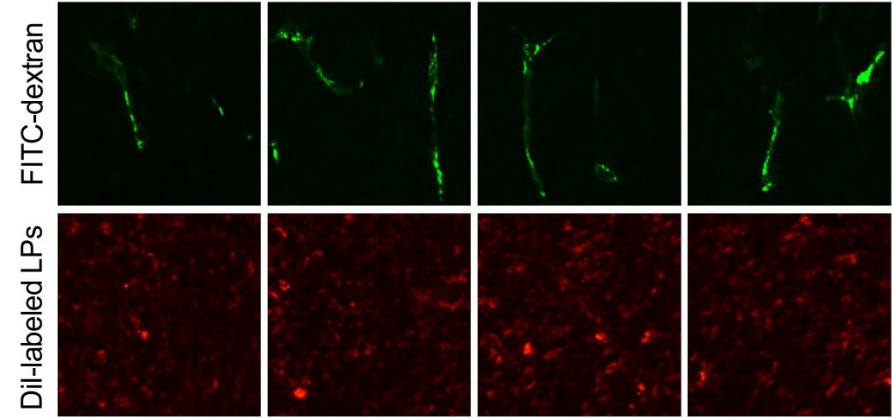

$\frac{0}{0}$
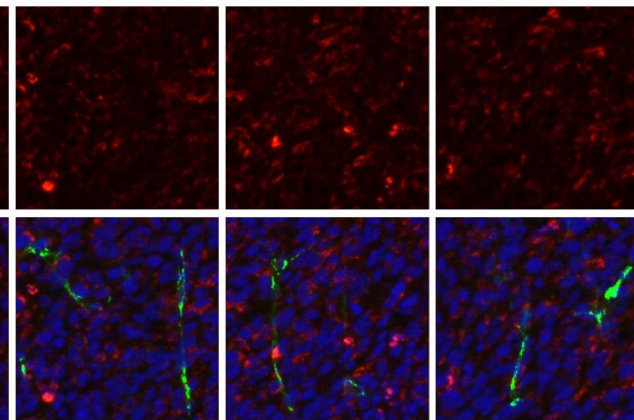

B16

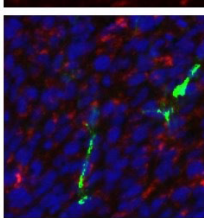

(
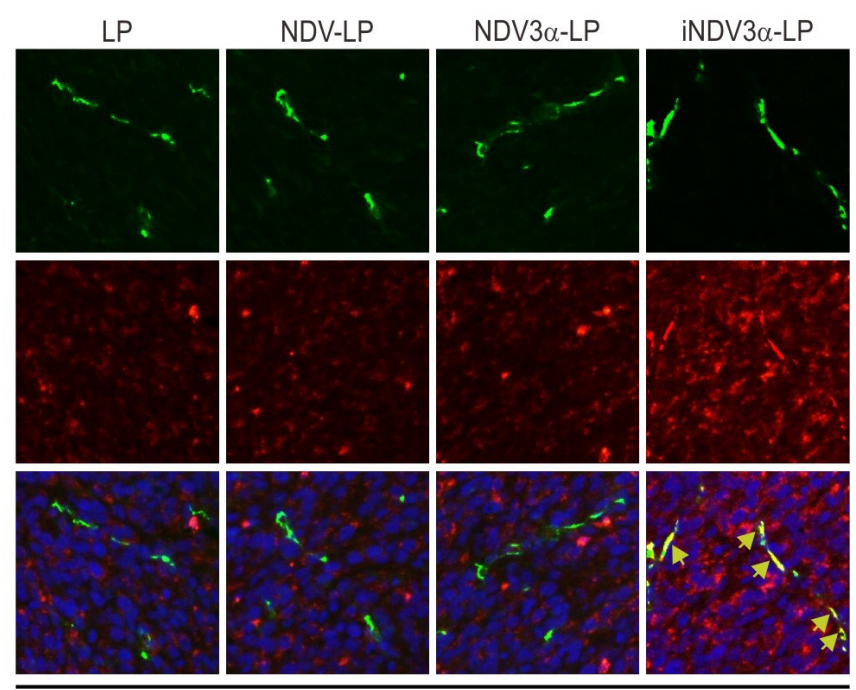

$4 \mathrm{~T} 1$

Figure 4 iNDV3 $\alpha$-LP targets to $\alpha v \beta 3$-expressing tumor cells and HUVECs and to tumor vasculature. (A) Immunoblotting detection of integrin $\alpha v \beta 3$ expression in B16, 4T1, and HUVECs. (B-D) confocal fluorescent images showing that FITCconjugated iNDV3 $\alpha$-LP (green, see also online supplemental figure S1F) binds to 4T1 (C) and HUVEC (D) cell membranes (Dil strained, red) but not to B16 (B); scale bar $=5 \mu \mathrm{m}$. (E) FCM analysis of the green fluorescence intensity on the cell surface, showing the representative images and MFI fold change of the B16 cells, 4T1 cells, and HUVECs treated with the indicated formulations. (F) Uptake analysis of $4 \mathrm{~T} 1$ cells by flow cytometry. (F) Confocal fluorescent images of B16 and 4T1 tumor sections, showing that only Dil-labeled iNDV3 $\alpha$-LP (red) specifically binds to FITC-dextran-marked blood vessels (green), which are merged as yellow (arrow). HUVECs, human umbilical vein endothelial cells; iNDV3 $\alpha$-LP, iRGD-liposome loaded with NDV expressing MIP- $3 \alpha$.

abdominal cavity of syngeneic mice to analyze the cell proliferation by FCM. The results show that the CD45negative CFSE-conjugated 4T1 or B16 cells proliferated for four generations, with an increased cell number observed in the LP group compared with that in the iNDV3 $\alpha-L P$, NDV3 $\alpha-L P$, and NDV-LP groups. However, 

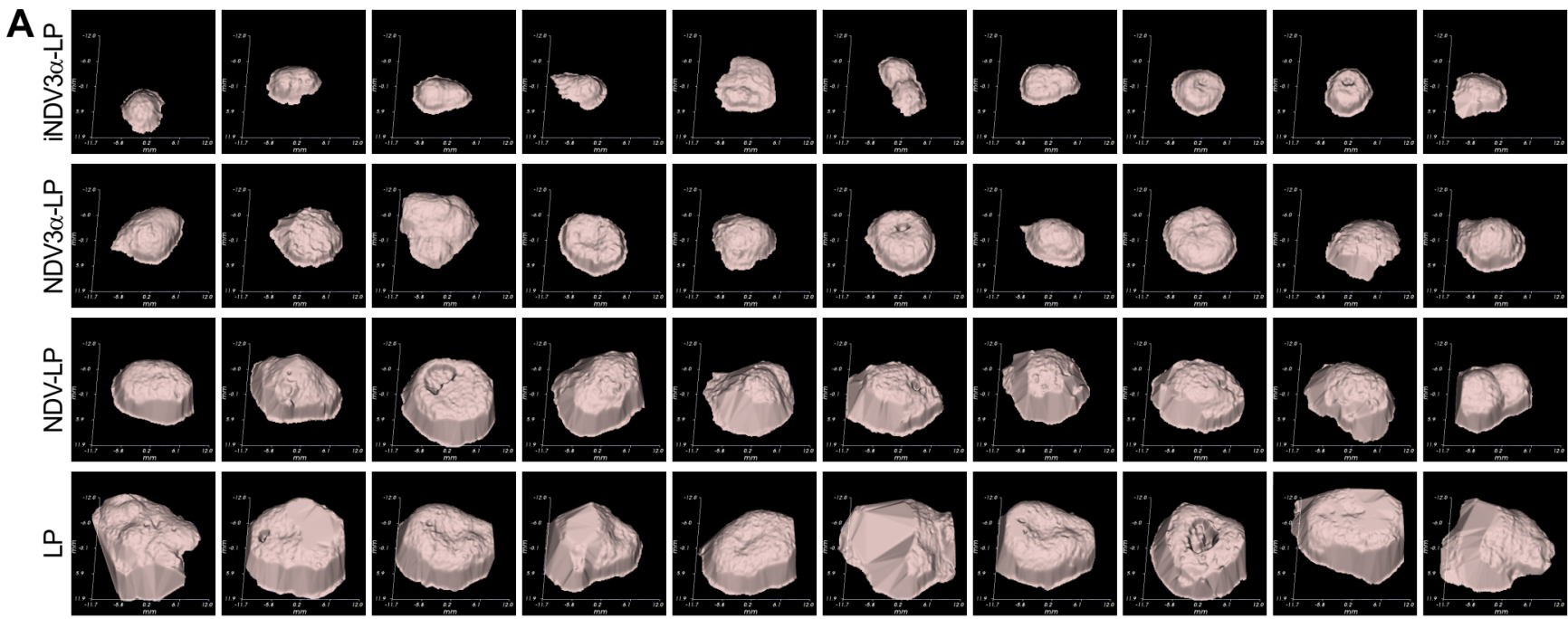

B

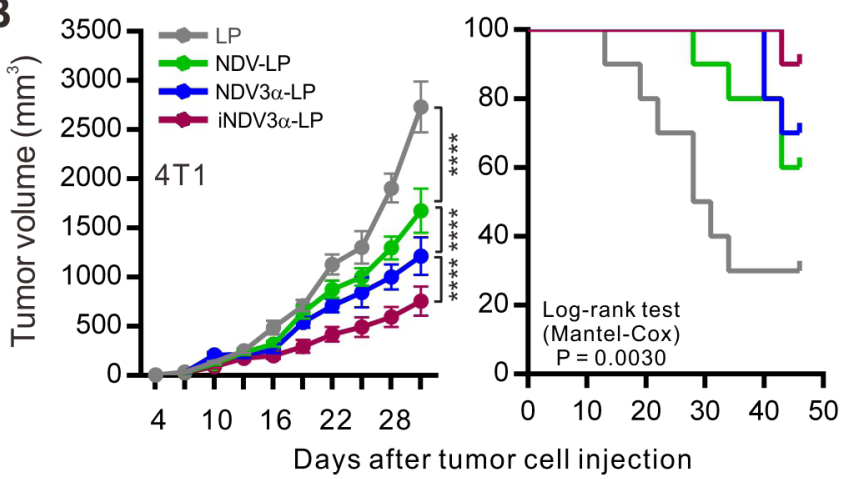

C

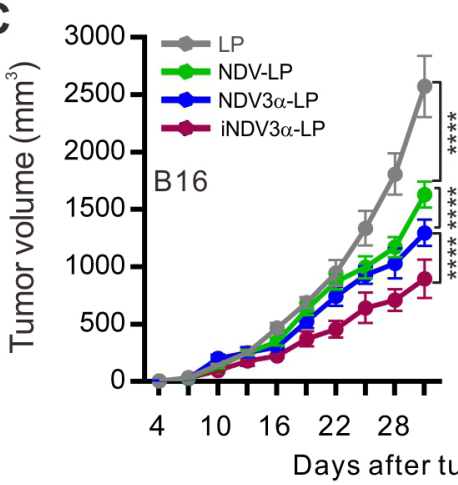

\begin{tabular}{|c|c|c|c|c|c|c|c|c|c|c|c|}
\hline \multirow{2}{*}{$\begin{array}{c}\text { Group } \\
4 T 1\end{array}$} & \multirow{2}{*}{$\begin{array}{c}\text { NDV-LP } \\
\text { ORR }\end{array}$} & \multirow{2}{*}{$\begin{array}{c}\text { NDV } 3 \alpha-L P \\
\text { ORR }\end{array}$} & \multicolumn{2}{|c|}{ iNDV3 $\alpha-L P$} & \multirow{2}{*}{ SI } & \multirow{2}{*}{$\begin{array}{c}\text { Group } \\
\text { B16 }\end{array}$} & \multirow{2}{*}{$\begin{array}{c}\text { NDV-LP } \\
\text { ORR }\end{array}$} & \multirow{2}{*}{$\begin{array}{c}\text { NDV } 3 \alpha-L P \\
\text { ORR }\end{array}$} & \multicolumn{2}{|c|}{ iNDV3 $\alpha-L P$} & \multirow{2}{*}{ SI } \\
\hline & & & ORR & ERR & & & & & ORR & ERR & \\
\hline Day 13 & 0.935 & 0.848 & 0.700 & 0.793 & 1.133 & Day 13 & 1.005 & 1.009 & 0.710 & 1.014 & 1.428 \\
\hline Day 16 & 0.660 & 0.534 & 0.417 & 0.352 & 0.844 & Day 16 & 0.752 & 0.647 & 0.476 & 0.487 & 1.021 \\
\hline Day 19 & 0.902 & 0.760 & 0.416 & 0.686 & 1.649 & Day 19 & 0.916 & 0.762 & 0.540 & 0.698 & 1.291 \\
\hline Day 22 & 0.776 & 0.627 & 0.371 & 0.486 & 1.312 & Day 22 & 0.908 & 0.783 & 0.481 & 0.710 & 1.477 \\
\hline Day 25 & 0.770 & 0.648 & 0.378 & 0.498 & 1.320 & Day 25 & 0.739 & 0.694 & 0.480 & 0.513 & 1.067 \\
\hline Day 28 & 0.681 & 0.525 & 0.312 & 0.358 & 1.146 & Day 28 & 0.651 & 0.569 & 0.393 & 0.370 & 0.942 \\
\hline Day 31 & 0.614 & 0.445 & 0.277 & 0.273 & 0.984 & Day 31 & 0.632 & 0.504 & 0.349 & 0.318 & 0.913 \\
\hline
\end{tabular}

Figure 5 iNDV3 $\alpha$-LP treatment induces specific antitumor effects. (A) Representative images of $4 T 1$ tumor masses treated with the indicated formulations on day 28 after tumor cell injection. (B and $C$ ) Data of the tumor volumes and survival rates of the tumor-bearing B16 and 4T1 mice treated with the indicated formulations at the indicated time points. (D) The synergistic indexes (SIs) calculated with the mean tumor volumes at the indicated time points in (B) and (C). ORR=mean tumor volume in the NDV-LP, NDV3 $\alpha$-LP, or iNDV3 $\alpha$-LP group/the mean tumor volume in the LP group; ERR=ORR of the NDV-LP group $\times$ ORR of NDV3 $\alpha$-LP; $\mathrm{SI}=\mathrm{ERR} / \mathrm{ORR}(\mathrm{ORR}<1)$ or ORR/ERR $(\mathrm{ORR}>1)$, where $\mathrm{Sl}>1$ indicates a synergistic effect. ERR, expected relative ratio; iNDV3 $\alpha$-LP, iRGD-liposome loaded with NDV expressing MIP-3 $\alpha$; NDV3 $\alpha$-LP, liposome loaded with NDV expressing MIP$3 \alpha$; NDV-LP, liposome loaded with single NDV; ORR, observed relative ratio.

cell proliferation was significantly inhibited, and the tumor cell number was markedly decreased in the iNDV3 $\alpha-L P$, NDV3 $\alpha-L P$, and NDV-LP groups, with the most significant decrease in the iNDV3 $\alpha$-LP group (figure 6C,D and online supplemental figure $S 1 G$ ). Additionally, the CD $45^{+}$immune cells from both 4T1 and B16 tumor tissues were gated and analyzed by FCM. $\mathrm{CD} 8^{+}$cells from
iNDV3 $\alpha$-LP-treated tumors expressed more active cytokines, such as IFN- $\gamma$ (figure 6E and online supplemental figure S2A,B), TNF- $\alpha$ (figure $6 \mathrm{~F}$ and online supplemental figure S2C,D), and IL-2 (figure 6G and online supplemental figure S2E,F). We also used the mean per cent of these immune cell subsets to calculate their SIs, most of which were $>1$ (figure 6H). These results also suggest that 

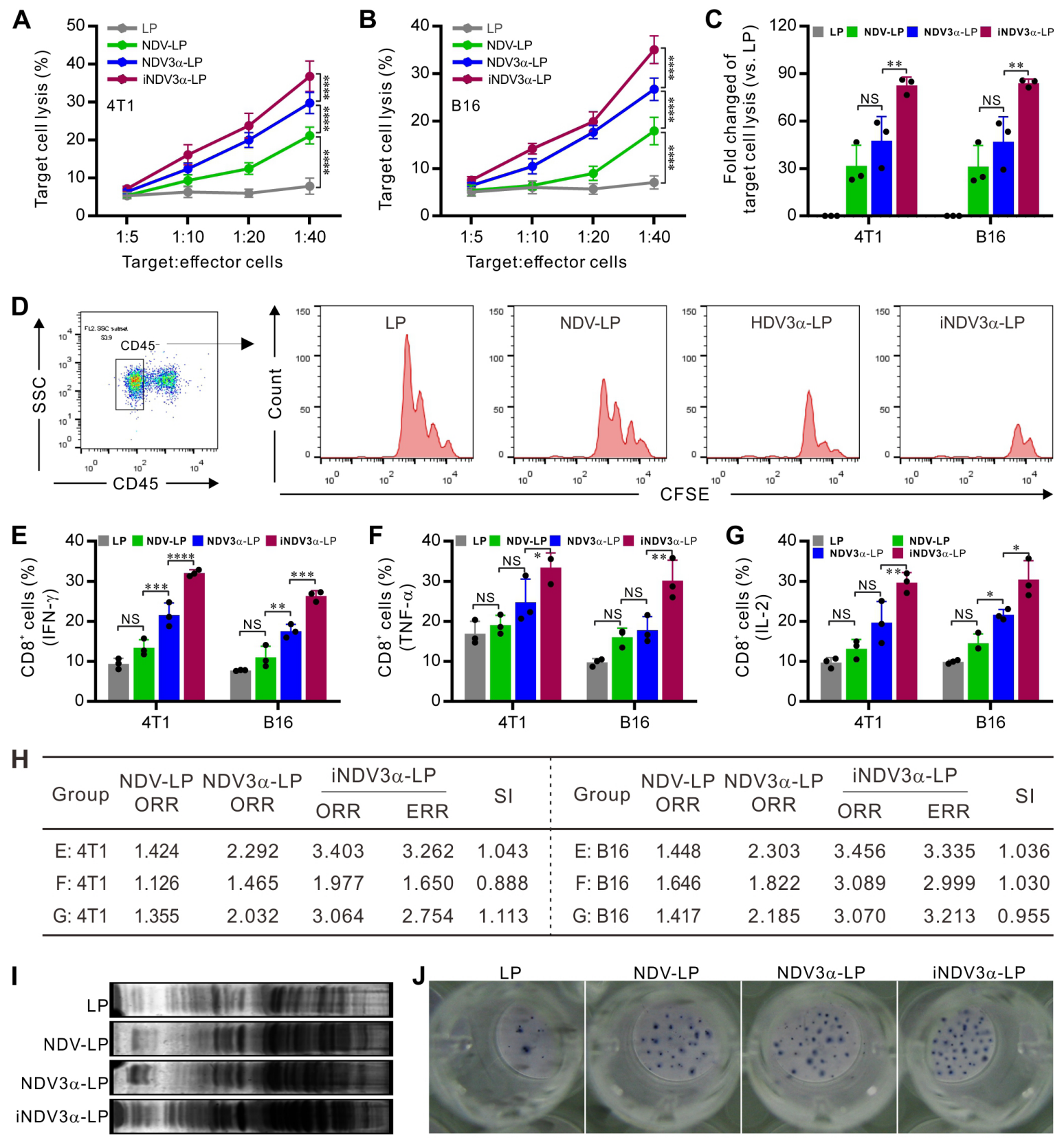

\begin{tabular}{lc|ccccccc}
\hline LP & \multirow{2}{*}{ SI } & Group & NDV-LP & NDV3 $\alpha-L P$ & \multicolumn{3}{c}{ iNDV3 $\alpha-L P$} & \\
\cline { 1 - 3 } & & & & ORR & ORR & ORR & ERR & SI \\
\hline 3.262 & 1.043 & E: B16 & 1.448 & 2.303 & 3.456 & 3.335 & 1.036 \\
1.650 & 0.888 & F: B16 & 1.646 & 1.822 & 3.089 & 2.999 & 1.030 \\
2.754 & 1.113 & G: B16 & 1.417 & 2.185 & 3.070 & 3.213 & 0.955 \\
\hline
\end{tabular}
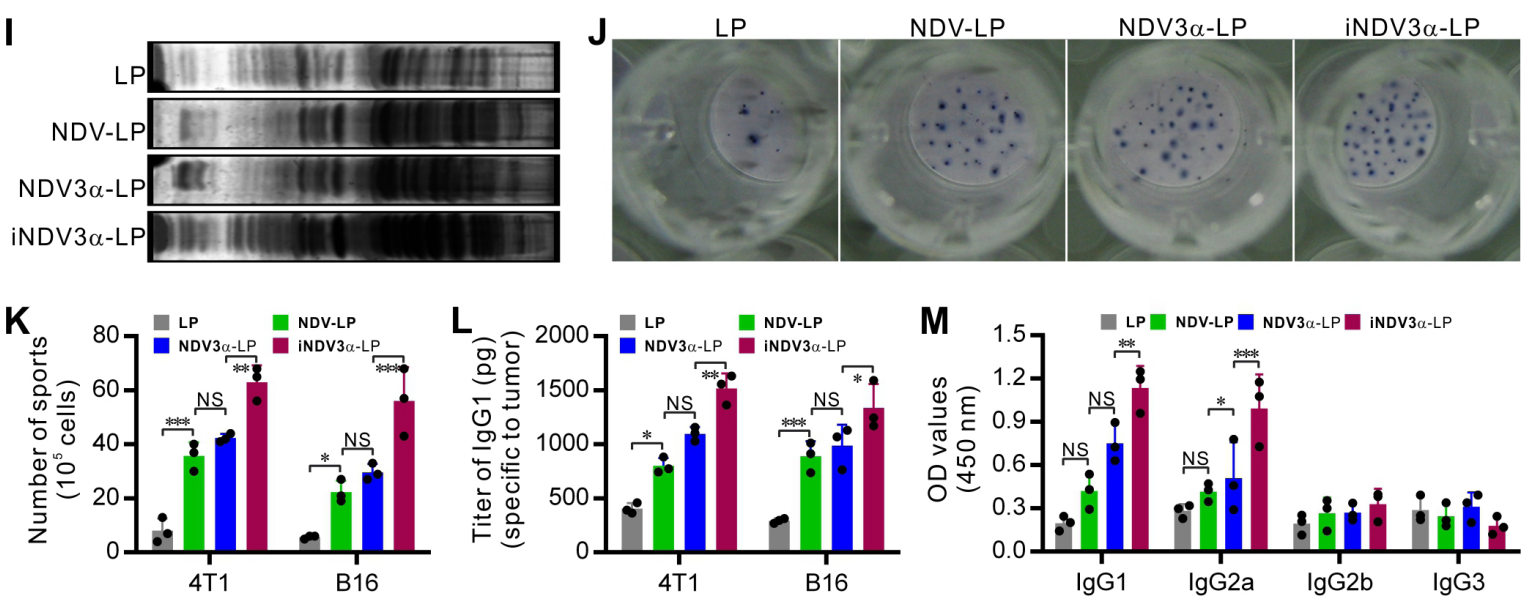

Figure 6 iNDV3 $\alpha$-LP treatment enhances antitumor immunity. (A and B) CTL response using B16 (A) and 4T1 (B) as target cells and the splenocytes from the mice treated with the indicated formulations as effector cells. (C and D) The in vivo CTL response was examined by injecting CFSE-labeled B16 (D) or 4T1 cells into the mouse abdomen treated with the indicated formulations to analyze the proliferation of $\mathrm{CD} 45^{-}$tumor cells by FCM and calculate the percentage of cell lysis (C, see also online supplemental figure S1). (E-G) The percentage of $C D 8^{+}$splenocytes secreting IFN- $\gamma(E)$, TNF- $\alpha(F)$, and IL-2 (G) from B16- or 4T1-bearing mice treated with the indicated formulations. $(H)$ The synergistic indexes (SIs) calculated with the mean percentage of $C D 8^{+}$cell subtypes in $(E-G)$. (I) Western blot detection of the tumor-specific IgG in the serum from mice treated with the indicated formulations. ( $\mathrm{J}$ and $\mathrm{K}$ ) ELISPOT detection of the splenocytes secreting IgG specific to B16 or 4T1 cells $(\mathrm{J})$ and the average number of IgG-secreting cells in $10^{5}$ splenocytes $(K)$. ( $L$ and $\left.M\right)$ The $\lg G$ titer in the serum from B16-bearing or 4T1-bearing mice treated with the indicated formulations (L) and the IgG subtypes (M). ELISPOT, enzyme-linked immunospot; iNDV3 $\alpha$-LP, iRGD-liposome loaded with NDV expressing MIP-3 $\alpha$. 
combination therapy induced by iNDV3 $\alpha$-LP provokes more tumor-specific cellular immunity in both $4 \mathrm{~T} 1$ and B16 tumor-bearing mice.

Moreover, we found that iNDV3 $\alpha$-LP treatment could induce stronger tumor-specific humoral immunity compared with the LP, NDV3 $\alpha$-LP, and NDV-LP groups. Compared with LP treatment, iNDV3 $\alpha$-LP, NDV3 $\alpha$-LP, and NDV-LP treatments showed significantly increased titers of IgG antibody both in 4T1-bearing and B16bearing mouse sera (figure 6I). Moreover, iNDV3 $\alpha$-LP treatment induced better tumor-specific humoral immunity compared with the other three groups, as confirmed by ELISPOT assay, in which more splenocytes secreting tumor-specific IgG were observed in the iNDV3 $\alpha$-LPtreated spleens (figure 6J-K). Consistently, quantitative analysis of IgG1 by ELISA also showed significantly increased IgG titers in the sera from mice treated with iNDV3 $\alpha$-LP, NDV3 $\alpha$-LP, and NDV-LP, with the highest titers observed in mice treated with iNDV3 $\alpha$-LP (figure 6L). Additionally, ELISA analysis showed that the significantly increased titers of IgG subsets were IgG1 and IgG2a (figure 6M). Taken together with the data shown in figure $6 \mathrm{E}-\mathrm{G}$, these results suggest that iNDV3 $\alpha-\mathrm{LP}$ promotes the induction of tumor-specific humoral immunity by stimulating both Th1 and Th2 immune responses.

\section{iNDV3 $\alpha$-LP inhibits tumor vascularization and modulates the tumor microenvironment}

Because iNDV3 $\alpha$-LP targets $\alpha v \beta 3$ on vascular endothelial cells, we next observed whether iNDV3 $\alpha$-LP treatment could inhibit tumor angiogenesis in 4T1 and B16 models. Angiogenesis within 4T1 and B16 tumor masses was observed on sections stained with an antibody against CD31, in which inhibition of angiogenesis was observed in iNDV3 $\alpha$-LP-treated sections (figure 7A). Moreover, compared with the LP-treated sections, the mean number of vessels per high-power field was significantly decreased in the iNDV3 $\alpha$-LP-treated, NDV3 $\alpha$-LP-treated, and NDVLP-treated sections, with the most significant decrease observed in the iNDV3 $\alpha$-LP-treated sections (figure 7B). Additionally, an alginate-bead encapsulation assay was adapted to quantify the angiogenesis by measuring the uptake of FITC-dextran into the tumor cell encapsulated alginate beads. ${ }^{15} 16$ Consistently, compared with LP treatment, iNDV3 $\alpha$-LP, NDV3 $\alpha$-LP, or NDV-LP treatment resulted in clear inhibition of angiogenesis in both 4T1-encapsulated and B16-encapsulated beards, but the most evident inhibition was found in the iNDV3 $\alpha$-LPtreated beads (figure 7C).

To investigate whether iNDV3 $\alpha$-LP treatment could modulate the immunosuppressive microenvironment, single cells were generated from 4T1 or B16 tumor masses. The $\mathrm{CD} 45^{+}$immune cells were gated out from the total cell population, and their subtypes, including IFN- $\gamma$-secreting $\mathrm{CD}^{+}$, myeloid-derived suppressor cells (MDSCs), regulatory $\mathrm{T}$ cells (Tregs), and tumor-associated macrophages (TAMs), were analyzed by FCM (figure 7D and online supplemental figure S3A-G). The results showed that the percentage of IFN- $\gamma$-expressing $\mathrm{CD}^{+}$lymphocytes was significantly increased in the iNDV3 $\alpha$-LP-treated tumor tissues (figure 7D-E and online supplemental figure S3A). However, the percentages of $\mathrm{CD}_{11 \mathrm{c}^{+}}$and $\mathrm{Gr}^{-1} \mathrm{1}^{+} \mathrm{MDSCs}$ (figure $7 \mathrm{~F}$ and online supplemental figure S3B,C), Foxp $3^{+}$and CD25 $5^{+}$Tregs (figure $7 \mathrm{G}$ and online supplemental figure S3E), and $\mathrm{F} 4 / 80^{+}$and $\mathrm{CD} 206^{+} \mathrm{M} 2$ TAMs (figure $7 \mathrm{H}$ and online supplemental figure S3 F-G) were significantly decreased in iNDV3 $\alpha$-LP-treated tumor tissues. Taken together with the results shown in figure $6 \mathrm{E}-\mathrm{G}$, these results suggest that the immunosuppressive microenvironment is effectively reversed by iNDV3 $\alpha$-LP treatment.

To understand whether iNDV3 $\alpha$-LP treatment can effectively inhibit tumor vascularization and modulate the tumor microenvironment, we used the previous data to calculate the SIs. As predicted, almost all SIs calculated from the data shown in figure 7 were $>1$, and some were even $>2$ (figure 7I). With the SIs shown in figure $6 \mathrm{H}$, which were calculated from the data shown in figure $6 \mathrm{E}-\mathrm{G}$, these findings also indicate that combination therapy induced by iNDV $3 \alpha-L P$ enhances inhibition of tumor vascularization and modulation of the tumor microenvironment.

\section{DISCUSSION}

Oncolytic immunotherapy is a paradigm-shifting treatment modality for cancers, which is primarily based on virus-mediated oncolysis and the subsequent induction of tumor-specific immune responses. Several lines of evidence from clinical trials of currently available oncolytic immunotherapeutic indicate that robust tumorspecific immunity leads to remarkable and long-term responses in a subset of patients. ${ }^{18225}$ In this study, we engineered a tumor neovasculature-targeted liposome iNDV3 $\alpha$-LP coated with iRGD, which specifically binds to the $\alpha v \beta 3$ receptor on endothelial cells in the tumor neovasculature. The DC chemokine gene MIP-3 $\alpha$ was encapsulated in the liposome iNDV3 $\alpha-\mathrm{LP}$, as this chemokine has been shown to promote systemic antitumor immunity in several murine tumor models. ${ }^{24}$ Successful construction of iNDV3 $\alpha$-LP and the three control liposomes, NDV3 $\alpha$-LP, NDV-LP, and LP, was evidenced by electron microscopy observation, laser-based analysis, and encapsulation-efficiency detection of NDVs. In contrast to the results of our previous study, which showed no significant difference in viral replication and tumor lysis between recombinant NDV3 $\alpha$ and wild-type NDV, iNDV3 $\alpha$-LP promoted greater viral replication and tumor and HUVEC lysis compared with NDV-LP, although NDV3 $\alpha-L P$ also induced significant virus replication and tumor and HUVEC lysis compared with NDV-LP. Detection of ICD-marker DAMP molecules also showed that iNDV3 $\alpha$-LP-treated B16 and 4T1 produced or secreted more DAMP molecules. As predicted, iNDV3 $\alpha$-LP could specifically bind to $\alpha \mathrm{v} \beta 3$-expressing $4 \mathrm{~T} 1$ and HUVECs in vitro and to the neovasculature in the tumor masses in vivo. Moreover, the tumor growth observed in B16 and 

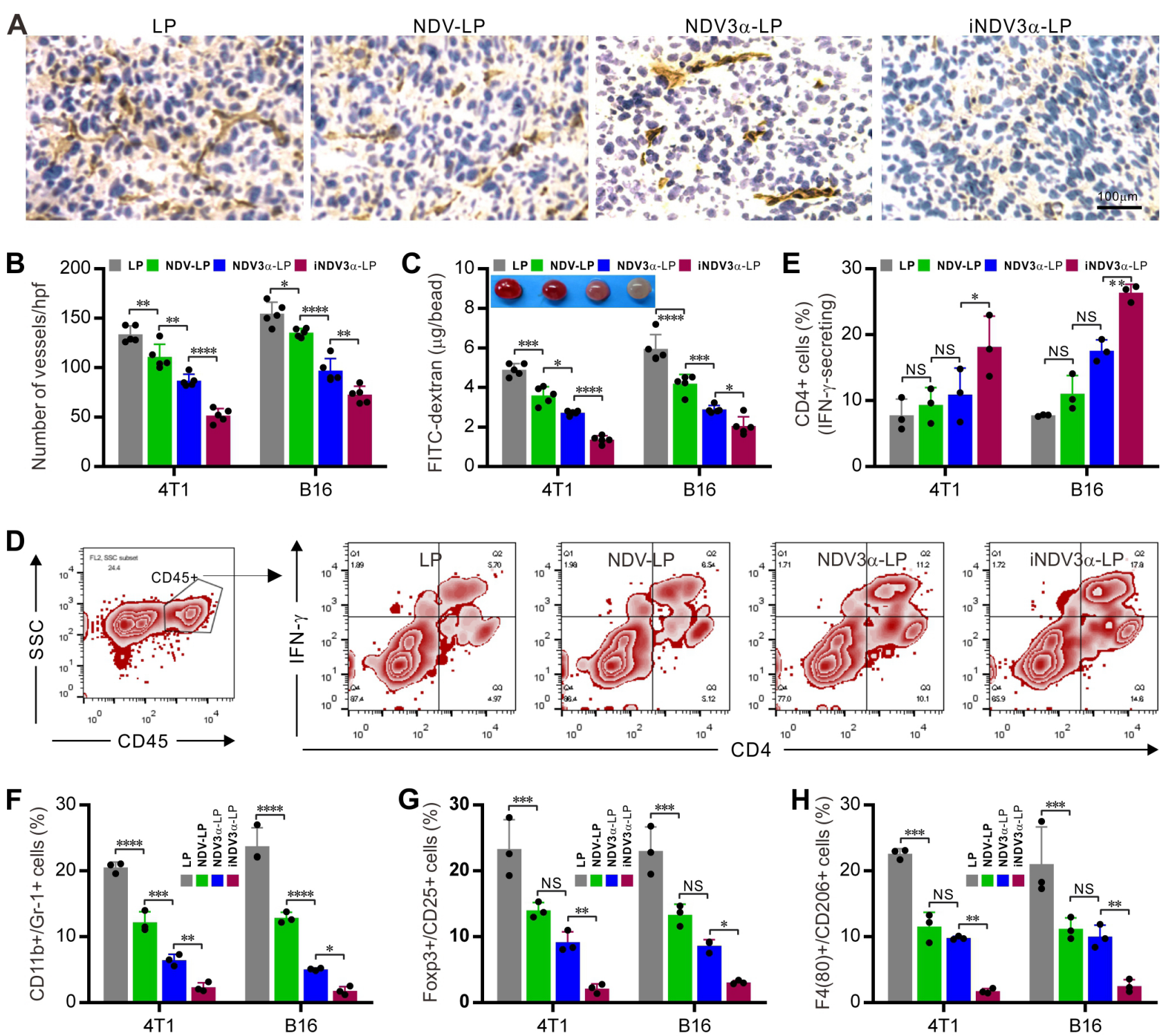

\begin{tabular}{|c|c|c|c|c|c|c|c|c|c|c|c|}
\hline \multirow{2}{*}{ Group } & \multirow{2}{*}{$\begin{array}{c}\text { NDV-LP } \\
\text { ORR }\end{array}$} & \multirow{2}{*}{$\begin{array}{l}\text { NDV } 3 \alpha-L P \\
\text { ORR }\end{array}$} & \multicolumn{2}{|c|}{ iNDV3 $\alpha$-LP } & \multirow{2}{*}{ SI } & \multirow{2}{*}{ Group } & \multirow{2}{*}{$\begin{array}{c}\text { NDV-LP } \\
\text { ORR }\end{array}$} & \multirow{2}{*}{$\begin{array}{c}\text { NDV } 3 \alpha-L P \\
\text { ORR }\end{array}$} & \multicolumn{2}{|c|}{ iNDV3 $3 \alpha-L P$} & \multirow{2}{*}{ SI } \\
\hline & & & ORR & ERR & & & & & ORR & ERR & \\
\hline B: $4 \mathrm{~T} 1$ & 0.828 & 0.650 & 0.386 & 0.538 & 1.396 & B: B16 & 0.876 & 0.627 & 0.470 & 0.550 & 1.170 \\
\hline $\mathrm{C}: 4 \mathrm{~T} 1$ & 0.733 & 0.555 & 0.278 & 0.407 & 1.463 & C: B16 & 0.704 & 0.486 & 0.345 & 0.342 & 0.991 \\
\hline $\mathrm{E}: 4 \mathrm{~T} 1$ & 1.200 & 1.170 & 1.866 & 1.404 & 1.186 & E: B16 & 1.417 & 1.179 & 1.866 & 1.671 & 1.116 \\
\hline $\mathrm{F}: 4 \mathrm{~T} 1$ & 0.594 & 0.315 & 0.114 & 0.187 & 1.634 & F: B16 & 0.541 & 0.214 & 0.076 & 1.643 & 1.518 \\
\hline G: $4 \mathrm{~T} 1$ & 0.600 & 0.392 & 0.134 & 0.235 & 2.578 & G: B16 & 0.579 & 0.374 & 0.134 & 0.217 & 1.616 \\
\hline $\mathrm{H}: 4 \mathrm{~T} 1$ & 0.510 & 0.434 & 0.078 & 0.221 & 2.844 & $\mathrm{H}: \mathrm{B} 16$ & 0.533 & 0.476 & 0.119 & 0.253 & 2.121 \\
\hline
\end{tabular}

Figure 7 iNDV3 $\alpha$-LP treatment inhibits tumor angiogenesis and reverses tumor immunosuppressive microenvironment. (A and B) Sections of frozen B16 tumor tissues from mice treated with the indicated formulations were stained with anti-CD31 antibody to show the tumor microvessels $(A)$ and determine the vessel density by counting the number of microvessels per high-power field (B). (C) FITC-dextran uptake in alginate beads encapsulated with $2 \times 10^{5}$ B16 or 4T1 cells treated with the indicated formulations. (D-H) $\mathrm{CD} 45^{+}$immune cells were gated from the total cell population from the tumor tissues treated with the indicated formulations. Representative flow cytometry images ( $D$, also see online supplemental figure S3), and the mean percentage of TILs with the following features in the B16-bearing or 4T1-bearing mice treated with the indicated formulations: IFN- $\gamma$-secreting CD4 ${ }^{+}$lymphocytes (E), CD11b and GR-1 double positive MDSCs (F), Poxp3 and CD25 double positive Tregs (G), and F4/80 and CD206 double positive M2-type tumor-associated macrophages (H). (I) The synergistic indexes (Sls) were calculated with the mean percentage of TIL cell subtypes in (E-H). iNDV3 $\alpha$-LP, iRGD-liposome loaded with NDV expressing MIP-3 $\alpha$; MDSCs, myeloid-derived suppressor cells; TILs, tumor-infiltrating lymphocytes; Tregs:, regulatory T cells. 
4T1 murine models was remarkably suppressed, and the survival was longer in iNDV3 $\alpha$-LP-treated mice. The mechanism study performed using several immunological techniques showed that iNDV3 $\alpha$-LP treatment induced the strongest tumor-specific cellular and humoral immunity of all tested liposomes. Surprisingly, iNDV3 $\alpha$-LP treatment could concurrently suppress tumor angiogenesis and reverse the tumor immune-suppressive microenvironment in both B16 and 4T1 model mice. Taken together, these data suggest that iNDV3 $\alpha$-LP that targets both tumor and its neovasculature is a novel highly efficient strategy for oncolytic immunotherapy.

It is generally accepted that oncolytic immunotherapy is not a 'magic bullet' for cancer therapy, and the activity of monotherapy is modest for most agents. ${ }^{31}$ To enhance antitumor activities, oncolytic immunotherapy combined with other treatment modalities, including immune checkpoint inhibitors, immune modulators, cancer vaccines, chemotherapy, radiation therapy, or even photothermal therapy by nanoparticles, has been explored in various preclinical and clinical trials. ${ }^{5} 7932$ However, oncolytic immunotherapy has various problems that still require further study. Therefore, novel combination strategies to redirect the dominant immune response toward cancer cells are warranted. The successful combination of T-VEC-expressing GM-CSF has proved that OVs have an additional advantage of delivering various transgenes to enhance antitumor activities. We have previously demonstrated that a recombinant oncolytic Newcastle virus expressing MIP-3 $\alpha$ (NDV3 $\alpha$ ) can remarkably promote systemic antitumor immunity in several murine models. ${ }^{24}$ In this study, we hypothesized that targeting recombinant NDV3 $\alpha$ to both the tumor and its neovasculature by encapsulation of NDV3 $\alpha$ in an iRGD-coated liposome (iNDV3 $\alpha$-LP) will induce combinatory anti-tumor effects. We considered that iNDV $3 \alpha$-LP will function to directly lyse both tumor and blood vessels and may additionally enhance the production of specific antitumor immunities as a result of MIP- $3 \alpha$ directing DCs to tumor masses and increasing the presentation of tumor antigen. Our results showed that iNDV3 $\alpha-L P$, but not NDV3 $\alpha$-LP, directly induced the lysis of both tumor cells and HUVECs. Moreover, iNDV3 $\alpha$-LP could also bind to tumor neovasculature in vivo and secrete DC chemokine MIP-3 $\alpha$. Therefore, the iNDV3 $\alpha$-LP treatment could function as a combination of tumor and vessel lysis, and MIP-3 $\alpha$ immunotherapy, NDV3 $\alpha$-LP treatment as tumor lysis and MIP- $3 \alpha$ immunotherapy, NDV-LP treatment as only tumor lysis, and LP treatment as control. In this study, we calculated the SIs using the tumor volume, tumor angiogenesis, and percentage of TIL subtypes to evaluate whether the iNDV3 $\alpha$-LP treatment induced combinatory antitumor activities. ${ }^{15}$ Our results show that iNDV3 $\alpha$-LP treatment induces significant lysis of both tumor and endothelial cells in vitro and inhibition of B16 and 4T1 tumor growth, as evidenced by SIs $>1$ at most time points. Moreover, we also found that most of the SIs calculated using the data of angiogenesis and TIL were $>1$ (figure 7I). Taken together, these data indicate that the iNDV3 $\alpha$-LP treatment strategy induces combinatory antitumor activities by concurrent lysis of tumor and endothelial cells, suppression of tumor angiogenesis, and systemic modulation of the tumor immunosuppressive microenvironment.

In our study, the results demonstrated that NDV-LP only reserved the function of wild-type NDV (tumor lysis and induction of immunogenic cell death), and NDV3 $\alpha$-LP added immune reinforcing function due to the recombinant MIP-3 $\alpha$ chemokine; however, iNDV3 $\alpha$-LP had all the functions of both NDV-LP and NDV3 $\alpha$-LP. Therefore, the therapy with iNDV $3 \alpha-\mathrm{LP}$ could be considered as a treatment modality that combines NDV-LP with NDV3 $\alpha$-LP. Herein, synergy index (SI) analysis, an accepted method in the literature, was applied to determine the synergistic effect induced by iNDV3 $\alpha$-LP. SI $>1$ indicates a synergistic effect. Unfortunately, the results indicated that some SI was less than 1, which mainly appeared in figures $5 \mathrm{D}$ and $6 \mathrm{H}$. With an in-depth analysis of the samples, we found that there were only 10 and 3 samples in the figures 5D and $6 \mathrm{H}$, respectively, and there were extreme values appearing in every time point when SI was less than 1 . We thought that the extreme values may be the major reason for $\mathrm{SI}<1$. Therefore, it is necessary to increase the number of samples to further certify whether iNDV3 $\alpha$-LP immunotherapy induced a really synergistic effect in future study.

The formation of neovasculature, or angiogenesis, is a necessary prerequisite for tumor generation and metastasis. Several lines of evidence have shown that certain OVs have the capability to selectively target the tumor neovasculature. For example, the vesicular stomatitis virus can cause thrombosis in the tumor neovessels by selective infection of endothelial cells in the tumor microenvironment. ${ }^{33}$ Herpes simplex virus (HSV) and vaccinia virus replicate more effectively in the tumor microenvironment with high levels of vascular endothelial growth factor and fibroblast growth factor. ${ }^{33-35}$ Thus, following successful infection, HSV and vaccinia virus can damage the tumor endothelium. ${ }^{33-35}$ Additionally, OVs can be genetically engineered to express antiangiogenic factors, such as VEGF inhibitors. ${ }^{36} 37$ Until now, no study has provided evidence of the ability of NDV to selectively damage the tumor endothelium. This phenomenon was proven by our results in that neither NDV3 $\alpha$-LP nor NDV-LP could target HUVECs in vitro or the tumor neovasculature in vivo and could not significantly lyse HUVECs in vitro. In contrast, iNDV3 $\alpha$-LP could bind to HUVECs in vitro and the tumor neovasculature in vivo and caused increased HUVEC lysis compared with the three other groups. Moreover, the most remarkable antiangiogenesis activities were found in the tumors from iNDV3 $\alpha$-LP-treated mice. Thus, we believe that the induction of the specific antitumor immunity by iNDV3 $\alpha$-LP may be of relevance to iRGD-directed binding and subsequent damage to the neovasculature in tumor-bearing mice. 
Immunogenic cell death (ICD) can activate DCs or other immune cells to trigger specific immunity against targeted cells. ${ }^{38}$ The induction of ICD changes 'cold' tumors to 'hot' tumors, which are more visible to the immune system, especially to DCs, which phagocytose the dying tumor cells, process and cross-present the tumor antigen and initiate tumor-specific immunity. ${ }^{39-41}$ During the ICD process, cancer cells induce endoplasmic reticulum (ER) stress, which is evidenced by the translocation of the ER-associated protein disulfide isomerase ERp57 from the ER lumen to the membranes of dying cells. ${ }^{42}$ During ER stress, CRT from the ER translocates to the cell surface, forming surface-expressed CRT (ecto-CRT). Additionally, cancer cells undergoing ICD express or secrete high levels of other DAMP molecules, such as extracellularly secreted ATP and passively released chromatinbinding protein HMGB1 ${ }^{43}$ The detection of these DAMP molecules helps to confirm existing ICD in tumor cells. In this study, compared with both NDV3 $\alpha$-LP- and NDVLP-treated cells, the results of western blot and ELISA showed remarkably increased levels of secreted HMGB1 (sHMGB1) in the supernatant of iNDV3 $\alpha$-LP-treated $\alpha v \beta 3$-expressing $4 \mathrm{~T} 1$ cells, but not in B16 cells. Although the sHMGB1 titer significantly increased in iNDV3 $\alpha$-LPtreated B16 cells, there were no significant differences among iNDV3 $\alpha$-LP-treated, NDV3 $\alpha$-LP-treated, and NDVLP-treated B16 cells. The results of ATP and ecto-CRT were similar to that of sHMGB1. Additionally, several parameters, including cell lysis, neovasculature-targeting, tumor volume, cellular and humoral immunity, and antiangiogenesis, were only superior in iNDV3 $\alpha$-LP-treated $4 \mathrm{~T} 1$ cells or mice. These data indicate that iNDV3 $\alpha-\mathrm{LP}$ treatment leads to increased production of ICD-related DAMPs, which suggests that the induction of better antitumor effects by iNDV3 $\alpha$-LP treatment is related to its neovasculature targeting the $\alpha v \beta 3$ positive endothelium.

\section{CONCLUSION}

In this study, we engineered an iRGD-liposome encapsulating the recombinant NDV, which expressed the DC chemokine MIP-3 $\alpha$ (named as iNDV3 $\alpha-\mathrm{LP}$ ), to explore its antitumor activities. The results show that iNDV3 $\alpha$-LP increased viral replication, tumor and HUVEC cell lysis, stronger tumor ICD, and a more remarkable antitumor immunity. The mechanism investigations show that iNDV3 $\alpha$-LP bound to $\alpha v \beta 3$-expressing tumor and HUVECs in vitro and to the neovasculature in the tumor masses in vivo. Additionally, iNDV3 $\alpha$-LP treatment triggered stronger tumor-specific cellular and humoral immunity. Moreover, iNDV3 $\alpha$-LP treatment significantly suppressed tumor angiogenesis and reversed the tumor immunosuppressive microenvironment. These findings offer a strong rationale for further clinical investigation into a combination strategy using multifunctional recombinant $\mathrm{OV}$ as a tool, such as the formulation iNDV3 $\alpha-\mathrm{LP}$ in this study.

\section{Author affiliations}

${ }^{1}$ Department of Breast and Thyroid Surgery, The Second Affiliated Hospital of Hainan Medical University; Key Laboratory of Tropical Translational Medicine of Ministry of Education \& Hainan Provincial Key Laboratory of Tropical Medicine, Hainan Medical University, Haikou, Hainan, China

${ }^{2}$ Department of Breast and Thyroid Surgery, Union Hospital, Tongji Medical College, Huazhong University of Science and Technology, Wuhan, Hubei, China ${ }^{3}$ Department of Respiratory Diseases, The First Affiliated Hospital of Hainan Medical University, Hainan Medical University, Haikou, Hainan, China

Contributors G-HT, F-YH, W-PZ, and LL designed experiments and analyzed and interpreted the data. J-YW, HC, S-ZD, F-YH, Y-YL, and C-CW performed the experiments. G-HT and F-YH analyzed the FCM data. W-PZ and LL offered some of the experiment resources and technical support. G-HT and F-YH directed the project and wrote the manuscript. All authors approved the final version of the manuscript. G-HT is the guarantor responsible for the overall content.

Funding This work was supported by the National Natural Science Foundation of China (81760634, 81860650 and 81960547, 82060639), and by the Hainan Provincial National Natural Science Foundation (2019RC217, 2019RC234 and ZDKJ202003). We would like to thank the Project supported by Hainan Province Clinical Medical Center.

Competing interests None declared.

Patient consent for publication Not applicable.

Provenance and peer review Not commissioned; externally peer reviewed.

Data availability statement Data are available on reasonable request.

Supplemental material This content has been supplied by the author(s). It has not been vetted by BMJ Publishing Group Limited (BMJ) and may not have been peer-reviewed. Any opinions or recommendations discussed are solely those of the author(s) and are not endorsed by BMJ. BMJ disclaims all liability and responsibility arising from any reliance placed on the content. Where the content includes any translated material, BMJ does not warrant the accuracy and reliability of the translations (including but not limited to local regulations, clinical guidelines, terminology, drug names and drug dosages), and is not responsible for any error and/or omissions arising from translation and adaptation or otherwise.

Open access This is an open access article distributed in accordance with the Creative Commons Attribution Non Commercial (CC BY-NC 4.0) license, which permits others to distribute, remix, adapt, build upon this work non-commercially, and license their derivative works on different terms, provided the original work is properly cited, appropriate credit is given, any changes made indicated, and the use is non-commercial. See http://creativecommons.org/licenses/by-nc/4.0/.

ORCID iD

Guang-Hong Tan http://orcid.org/0000-0002-6406-0331

\section{REFERENCES}

1 Fukuhara $\mathrm{H}$, Ino $\mathrm{Y}$, Todo T. Oncolytic virus therapy: a new era of cancer treatment at dawn. Cancer Sci 2016;107:1373-9.

2 Ehrlich M, Bacharach E. Oncolytic virotherapy: the cancer cell side. Cancers 2021;13. doi:10.3390/cancers13050939. [Epub ahead of print: 24 Feb 2021].

3 Fountzilas C, Patel S, Mahalingam D. Review: oncolytic virotherapy, updates and future directions. Oncotarget 2017;8:102617-39.

4 Kelly E, Russell SJ. History of oncolytic viruses: genesis to genetic engineering. Mol Ther 2007;15:651-9.

5 Chiocca EA, Rabkin SD. Oncolytic viruses and their application to cancer immunotherapy. Cancer Immunol Res 2014;2:295-300.

6 Aurelian L. Oncolytic viruses as immunotherapy: progress and remaining challenges. Onco Targets Ther 2016;9:2627-37.

7 Bartlett DL, Liu Z, Sathaiah M, et al. Oncolytic viruses as therapeutic cancer vaccines. Mol Cancer 2013;12:103.

8 Luo Y, Lin C, Zou Y, et al. Tumor-targeting oncolytic virus elicits potent immunotherapeutic vaccine responses to tumor antigens. Oncoimmunology 2020;9:1726168.

9 Russell SJ, Barber GN. Oncolytic viruses as Antigen-Agnostic cancer vaccines. Cancer Cell 2018;33:599-605.

10 Cassidy T, Craig M. Determinants of combination GM-CSF immunotherapy and oncolytic virotherapy success identified through in silico treatment personalization. PLoS Comput Biol 2019;15:e1007495. 
11 Hamid O, Ismail R, Puzanov I. Intratumoral Immunotherapy-Update 2019. Oncologist 2020;25:e423-38.

12 Fleeman N, Bagust A, Boland A, et al. Talimogene Laherparepvec for treating metastatic melanoma: an evidence review group perspective of a NICE single technology appraisal. Pharmacoeconomics 2017;35:1035-46.

13 Andtbacka RHI, Kaufman HL, Collichio F, et al. Talimogene Laherparepvec improves durable response rate in patients with advanced melanoma. J Clin Oncol 2015;33:2780-8.

14 Lai V, Neshat SY, Rakoski A, et al. Drug delivery strategies in maximizing anti-angiogenesis and anti-tumor immunity. Adv Drug Deliv Rev 2021;179:113920.

15 Tan G-H, Tian L, Wei Y-Q, et al. Combination of low-dose cisplatin and recombinant xenogeneic endoglin as a vaccine induces synergistic antitumor activities. Int J Cancer 2004;112:701-6.

16 Tan G-H, Wei Y-Q, Tian L, et al. Active immunotherapy of tumors with a recombinant xenogeneic endoglin as a model antigen. Eur $J$ Immunol 2004;34:2012-21.

17 Desgrosellier JS, Cheresh DA. Integrins in cancer: biological implications and therapeutic opportunities. Nat Rev Cancer 2010;10:9-22.

18 Wang J, Wang H, Li J, et al. iRGD-Decorated polymeric nanoparticles for the efficient delivery of vandetanib to hepatocellular carcinoma: preparation and in vitro and in vivo evaluation. ACS Appl Mater Interfaces 2016;8:19228-37.

19 Lo JH, Hao L, Muzumdar MD, et al. iRGD-guided tumor-penetrating nanocomplexes for therapeutic siRNA delivery to pancreatic cancer. Mol Cancer Ther 2018;17:2377-88.

20 Yan F, Wu H, Liu H, et al. Molecular imaging-guided photothermal/ photodynamic therapy against tumor by iRGD-modified indocyanine green nanoparticles. J Control Release 2016;224:217-28.

21 Sugahara KN, Teesalu T, Karmali PP, et al. Tissue-penetrating delivery of compounds and nanoparticles into tumors. Cancer Cell 2009;16:510-20.

22 Wang K, Zhang X, Liu Y, et al. Tumor penetrability and antiangiogenesis using iRGD-mediated delivery of doxorubicin-polymer conjugates. Biomaterials 2014;35:8735-47.

23 Sugahara KN, Teesalu T, Karmali PP, et al. Coadministration of a tumor-penetrating peptide enhances the efficacy of cancer drugs. Science 2010;328:1031-5.

24 Huang F-Y, Wang J-Y, Dai S-Z, et al. A recombinant oncolytic Newcastle virus expressing MIP- $3 \alpha$ promotes systemic antitumor immunity. J Immunother Cancer 2020;8:e000330.

25 Zamarin D, Holmgaard RB, Ricca J, et al. Intratumoral modulation of the inducible co-stimulator ICOS by recombinant oncolytic virus promotes systemic anti-tumour immunity. Nat Commun 2017;8:14340.

26 Wang $\mathrm{Y}$, Huang $\mathrm{H}$, Zou $\mathrm{H}$, et al. Liposome encapsulation of oncolytic virus $\mathrm{M} 1$ to reduce immunogenicity and immune clearance in vivo. Mol Pharm 2019;16:779-85.
27 Huang F-ying, Huang F-ru, Chen B, et al. Microencapsulation of tumor lysates and live cell engineering with MIP-3 $\alpha$ as an effective vaccine. Biomaterials 2015;53:554-65.

28 Huang F-Y, Lei J, Sun Y, et al. Induction of enhanced immunogenic cell death through ultrasound-controlled release of doxorubicin by liposome-microbubble complexes. Oncoimmunology 2018;7:e1446720.

29 Kepp O, Senovilla L, Vitale I, et al. Consensus guidelines for the detection of immunogenic cell death. Oncoimmunology 2014;3:e955691.

30 Bommareddy PK, Zloza A, Rabkin SD, et al. Oncolytic virus immunotherapy induces immunogenic cell death and overcomes sting deficiency in melanoma. Oncoimmunology 2019;8:1591875.

31 Sharma P, Hu-Lieskovan S, Wargo JA, et al. Primary, adaptive, and acquired resistance to cancer immunotherapy. Cell 2017;168:707-23.

32 Filley AC, Dey M, System I. Immune system, friend or foe of oncolytic virotherapy? Front Oncol 2017;7:106.

33 Breitbach CJ, De Silva NS, Falls TJ, et al. Targeting tumor vasculature with an oncolytic virus. Mol Ther 2011;19:886-94.

34 Breitbach CJ, Arulanandam R, De Silva N, et al. Oncolytic vaccinia virus disrupts tumor-associated vasculature in humans. Cancer Res 2013;73:1265-75.

35 Benencia F, Courreges MC, Conejo-García JR, et al. Oncolytic HSV exerts direct antiangiogenic activity in ovarian carcinoma. Hum Gene Ther 2005;16:765-78.

36 Zhang Z, Zou W, Wang J, et al. Suppression of tumor growth by oncolytic adenovirus-mediated delivery of an antiangiogenic gene, soluble Flt-1. Mol Ther 2005;11:553-62.

37 Gholami S, Marano A, Chen NG, et al. A novel vaccinia virus with dual oncolytic and anti-angiogenic therapeutic effects against triple-negative breast cancer. Breast Cancer Res Treat 2014;148:489-99.

38 Ladoire S, Enot D, Andre F, et al. Immunogenic cell death-related biomarkers: impact on the survival of breast cancer patients after adjuvant chemotherapy. Oncoimmunology 2016;5:e1082706.

39 Vacchelli E, Ma Y, Baracco EE, et al. Chemotherapy-induced antitumor immunity requires formyl peptide receptor 1 . Science 2015;350:972-8

40 Zhao X, Yang K, Zhao R, et al. Inducing enhanced immunogenic cell death with nanocarrier-based drug delivery systems for pancreatic cancer therapy. Biomaterials 2016;102:187-97.

41 Obeid M, Tesniere A, Ghiringhelli F, et al. Calreticulin exposure dictates the immunogenicity of cancer cell death. Nat Med 2007;13:54-61

42 Obeid M. ERP57 membrane translocation dictates the immunogenicity of tumor cell death by controlling the membrane translocation of calreticulin. J Immunol 2008;181:2533-43.

43 Alzeibak R, Mishchenko TA, Shilyagina NY, et al. Targeting immunogenic cancer cell death by photodynamic therapy: past, present and future. J Immunother Cancer 2021;9:e001926. 